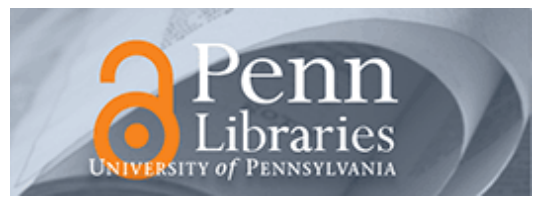

University of Pennsylvania

ScholarlyCommons

Finance Papers

Wharton Faculty Research

6-19-2016

\title{
Signaling to Partially Informed Investors in the Newsvendor Model
}

William Schmidt

Vishal Gaur

Richard Lai

Ananth Raman

Follow this and additional works at: https://repository.upenn.edu/fnce_papers

Part of the Finance and Financial Management Commons

\section{Recommended Citation}

Schmidt, W., Gaur, V., Lai, R., \& Raman, A. (2016). Signaling to Partially Informed Investors in the Newsvendor Model. Production and Operations Management, 24 (3), 383-401. http://dx.doi.org/10.1111/ poms. 12245

This paper is posted at ScholarlyCommons. https://repository.upenn.edu/fnce_papers/63

For more information, please contact repository@pobox.upenn.edu. 


\title{
Signaling to Partially Informed Investors in the Newsvendor Model
}

\author{
Abstract \\ We analyze a signaling game between the manager of a firm and an investor in the firm. The manager has \\ private information about the firm's demand and cares about the short-term stock price assigned by the \\ investor. Previous research has shown that under continuous decision choices and the Intuitive Criterion \\ refinement, the least-cost separating equilibrium will result, in which a low-quality firm chooses its optimal \\ capacity and a high-quality firm over-invests in order to signal its quality to investors. We build on this \\ research by showing the existence of pooling outcomes in which low-quality firms over-invest and high- \\ quality firms under-invest so as to provide identical signals to investors. The pooling equilibrium is \\ practically appealing because it yields a Pareto improvement compared to the least-cost separating \\ equilibrium. Distinguishing features of our analysis are that: (i) we allow the capacity decision to have \\ either discrete or continuous support, and (ii) we allow beliefs to be refined based on either the \\ Undefeated refinement or the Intuitive Criterion refinement. We find that the newsvendor model \\ parameters impact the likelihood of a pooling outcome, and this impact changes in both sign and \\ magnitude depending on which refinement is used. \\ Disciplines \\ Business | Finance and Financial Management
}




\section{Signaling to Partially Informed Investors in the Newsvendor Model}

Article in Production and Operations Management · February 2012

DOI: $10.2139 /$ ssrn.1815207

CITATIONS

4

4 authors, including:

\section{Vishal Gaur}

Cornell University

46 PUBLICATIONS 1,048 CITATIONS

SEE PROFILE
READS

178

Some of the authors of this publication are also working on these related projects: 
H A R VAR D

\section{Signaling to Partially Informed Investors in the Newsvendor Model}

William Schmidt

Vishal Gaur

Richard Lai

Ananth Raman

\section{Working Paper}

11-105

February 6, 2012 


\title{
Signaling to Partially Informed Investors in the Newsvendor Model
}

\author{
William Schmidt* $\quad$ Vishal Gaur $†$ Richard Lai $;$ Ananth Raman ${ }^{\S}$
}

February 6, 2012

\begin{abstract}
We investigate a phenomenon in which firms may attempt to influence their market valuation by choosing an inventory stocking quantity which does not optimize expected profits. We employ the newsvendor model within a signaling game to examine a relatively common scenario in which the firm's equity holder has incomplete information concerning the demand for the firm's product. We apply a perfect Bayesian equilibrium solution and identify ranges of model parameters where the firm's stocking quantity decision does not maximize expected profits. This includes instances in which a firm facing high demand chooses a lower stocking quantity than that which would optimize expected profits and a firm facing low demand chooses a higher stocking quantity than that which would optimize expected profits. This result contrasts with prior research, which has shown that when equity holders have incomplete information about the quality of the firm's opportunities, high quality firms will consistently overinvest and low quality firms will invest to optimize expected profits. We show an extreme example of this behavior in which a high demand firm chooses that stocking quantity which would have been optimal under complete information for a low demand firm.
\end{abstract}

\footnotetext{
*Harvard Business School, Soldiers Field Park, Boston MA 02163. E-mail: wschmidt@hbs.edu.

${ }^{\dagger}$ The Johnson School, Cornell University, Sage Hall, Ithaca NY 14850. E-mail: vg77@cornell.edu.

${ }^{\ddagger}$ E-mail: lairk@wharton.upenn.edu.

${ }^{\S}$ Harvard Business School, Soldiers Field Park, Boston MA 02163. E-mail: araman@hbs.edu.
} 


\section{Introduction}

We investigate the effect of short-term objectives and incomplete information on a firm's operational decisions. We incorporate the newsvendor model using discrete stocking quantities into a signaling game and identify the circumstances under which the firm's choice of stocking quantity purposefully does not maximize expected profits. Our focus is on the relatively common scenario in which a firm's equity holder (him) has less information than the firm (her) concerning the quality of demand for the firm's product. We apply a perfect Bayesian equilibrium (PBE) solution to characterize the model parameters in which the firm will under-stock, stock optimally or over-stock compared to the stocking quantity that optimizes expected profit.

The model accounts for two elements that are common in agency relationships - the external equity holder possesses incomplete information about the firm's prospects and the firm derives some utility from its short-term valuation (short-termism). The literature acknowledges that external investors can have information about a firm's operations that is inferior to the firm's information (Berle and Means 1932, Stein 1988). Information asymmetry is incorporated into our model by allowing the firm to know the distribution of random demand for its product while the equity holder knows only that the distribution of random demand comes from a set of possible distributions. Given this information asymmetry, the short-term valuation of the firm by the equity holder may differ from the true value of the firm.

Short-termism is captured in the model through a firm objective function that is a linear combination of both her short-term and long-term valuation. In practice, firm decision makers are cognizant of and manage the firm's short-term valuation (Jensen and Meckling 1976, Narayanan 1985). Managers may exhibit short-termism for a variety of reasons, including a desire to raise capital in a secondary offering, to dispose of personal shares in the firm at an attractive price, to prevent takeovers, or to burnish their reputation and careers (Jensen 1986, Stein 1988, Holmstrom 1999, Stein 2003). Practitioners have also acknowledged that firms accommodate long-term objectives in the face of short-term pressures. For example, Barton (2011) argues that the "mania over quarterly earnings consumes extraordinary amounts of senior [managers'] time and attention," and expresses dismay at "quarterly capitalism" (in which firms are unduly influenced by short-term pressures). Rappaport (2004) acknowledges that "[t]o meet Wall Street expectations, managers make decisions to increase short-term earnings - even at the expense of long-term shareholder value." Focusing 
on short-term performance at the expense of long-term performance does not appear to be a rare occurrence. In a survey of over 400 financial executives, Graham et al. (2005) found that over $78 \%$ would give up economic value in order to hit a short-term earnings target and $55 \%$ would delay initiating a project with a very positive net present value.

In our model, the firm's choice of order quantity provides a noisy signal to the equity holder which may mitigate the information asymmetry between the firm and the equity holder. We capture the noise of this signal by allowing orders to be placed only in multiples of a fixed quantity. This type of restriction is common in practice, where the fixed quantity may represent a container load, a pallet, case pack size or a production batch (Nahmias 2001). This is a critical difference between our model and the prior literature.

The prior literature has shown that under short-termism and information asymmetry concerning the quality of the firm's prospects, the resulting unique PBE is the least cost separating PBE in which type $\tau_{H}$ firms over-invest compared to their long-term optimal decisions while and type $\tau_{L}$ firms invest optimally (Bebchuk and Stole 1993, Lai et al. 2011b). These models do not address the possibility of firm under-investment because they assume a continuous decision space and they focus exclusively on the Intuitive Criterion refinement, as defined in Cho and Kreps (1987). In contrast, we show that when the stocking quantity choice is discrete, (1) pooling equilibria exist, (2) in some cases separating equilibria do not exist at all, (3) in many cases the pooling equilibria survive the Intuitive Criterion refinement, (4) if more than one pooling PBE exist then at least one survives the Undefeated refinement, and (5) if more than one PBE survives the Undefeated refinement, there is a unique lexicographically maximum sequential equilibrium (LMSE) from this set of PBE. Our model provides analytical support for the abundant anecdotal evidence that superior firms will knowingly under-invest in capacity under certain circumstances.

The information asymmetry in our model can be generalized beyond product demand to other situations such as the firm having better insight into the effectiveness of an emerging technology, the value of a new supply chain configuration, or the potential size of a new market. While we utilize the newsvendor model in the context of an inventory stocking decision, our model and analysis are easily generalized to a wide range of project investment decisions that a firm may encounter. The newsvendor model is merely a framework for making a capacity investment decision in the face of uncertainty. In this respect, our model can be effectively applied regardless of whether the decision 
is about inventory or some other type of capacity investment, including plant expansions, capital expenditures, and contracting for production inputs.

\section{Literature Review}

Our model and analysis leverage the literature dealing with investment decisions in the presence of short-term managerial objectives and incomplete information. We contribute to the literature by showing why a newsvendor may choose suboptimal inventory levels. We also add to the relatively sparse research linking the operations management literature on optimal inventory management to the literature on signaling games, which has been more commonly employed in finance and economics.

While typically employed in finance and economics, signaling game theory and Bayesian equilibrium solutions concepts have been utilized to explore a wide range of research questions in which there are multiple participants and asymmetric information among them (Spence 1974, Grossman 1981, Milgrom and Roberts 1986, Aghion and Bolton 1987, Grinblatt and Hwang 1989, Cramton and Tracy 1992, Chen et al. 2008). Our research applies specifically to the literature on a firm's capacity decisions in the presence of short-term managerial objectives and asymmetric information between the firm and its external investor. This literature can be classified into two categories one focused on information asymmetry concerning the amount of the investment made by the firm and the other focused on information asymmetry concerning the quality of the firm's investment opportunity. In the former category, Stein (1989) shows that firms will under invest in projects when external investors cannot observe the amount of the firm's investment.

In the latter category, Bebchuk and Stole (1993) demonstrate that a separating equilibrium will exist when external investors cannot observe the quality of the firm's investment opportunity. Similarly, Lai et al. (2011b) show that with continuous stocking quantities firms will over-stock in order to perfectly identify the quality of their demand prospects to external investors and that the resulting separating equilibrium is the only equilibrium which survives the Intuitive Criterion refinement. We contribute to this literature by exploring the impact of discrete stocking quantities, a common occurrence in capacity investment decisions, when there is information asymmetry on the quality of the firm's opportunity. We show that not only may a pooling equilibrium exist which survives the Intuitive Criterion refinement, but a separating equilibrium may not exist at all. 
Several researchers have examined circumstances in which managers may make operational decisions that do not maximize expected profits, either due to bias, complex environments, or private incentives. A body of experimental studies has identified that decision makers may deviate from the expected-profit-maximizing order quantity due to some sort of decision bias, including anchoring, demand chasing, and inventory error minimization (Schweitzer and Cachon 2000, Bolton and Katok 2008, Bostian et al. 2008, Kremer et al. 2010).

In addition to the literature involving experimental methods, Deshpande et al. (2003) and van Donselaar et al. (2010) use large sample observational data and provide empirical evidence that decisions in practice may differ from model-based rules. Lai (2006) utilizes a natural experiment involving a change in fiscal year end reporting cycles to show that firms may modify their inventory policies in an effort to influence the firm's valuation in the stock market. Using a two-period analytical model, Lai et al. (2011a) show that in order to improve their short-term valuation, firms may utilize channel stuffing to inflate their reported sales in the first period and signal higher demand in the second period. While this model shows that under-investment in inventory may occur, it is due to management trying to optimize expected firm profits by choosing an inventory stocking level that accounts for the cost of channel-stuffing. In our model, on the other hand, management consciously sets an inventory stocking level that they know is sub-optimal for expected profits. Lai et al. (2011b) develop a model in which firms also over-stock in order to reveal their demand prospects to external investors. The authors then explore contracts which can restore supply chain efficiency. We build off of this stream of literature by exploring the circumstances under which short-termism, information asymmetry, and discrete stocking quantities influence high quality firms to under-stock, and correspondingly, low quality firms to over-stock.

Finally, we seek to add to the small but growing use of signaling game theory in the operations management literature. While game theory has been employed extensively to research topics on supply chain coordination, there are relatively few applications specifically of signaling game theory in the operations management literature. Of those papers that do use signaling models, most of them explore how information is shared across the members of a supply chain. Islegen and Plambeck (2007) study capacity investments by a manufacturer and its supplier and characterize the conditions for a separating PBE when the manufacturer has superior demand information. Debo and Veeraraghavan (2010) explore how firms may use a combination of prices and congestion to 
signal quality to consumers. Anand and Goyal (2009) investigate how information is shared within a supply chain that consists of horizontal competitors and a common supplier. Allon and Bassamboo (2011) examine the information that retailers disclose to customers on product availability. We seek to expand on this literature by providing an important application of signaling game theory to the problem of inventory management in the face of an external equity holder. In addition, we introduce an application of the Undefeated refinement from Mailath et al. (1993), which to our knowledge has not been applied in the operations management literature.

\section{Model}

We analyze a signaling game with two players, $\mathrm{N}$ and $\mathrm{E}$, and two time periods. Player $\mathrm{N}$ is a newsvendor firm (she/her) and player $\mathrm{E}$ is an equity holder (he/him). Period 1 represents the short term and period 2 represents the long term. The players move sequentially under incomplete information. The set up for the game and the sequence of events are as follows.

The firm seeks to maximize her expected utility by choosing a stocking quantity $\eta$ to serve random demand. She is a price-taker in her product market, and has a purchase $\operatorname{cost} c$, selling price $r$, and salvage value $s$ of unsold inventory, where $r>c>s$. The firm can be of two types, $\tau_{L}$ and $\tau_{H}$, that differ only in the probability distribution of demand. Let $g(\tau), \tau \in T=\left\{\tau_{L}, \tau_{H}\right\}$ be the probability by which nature chooses the type of the firm, and let $f_{\tau}(\cdot)$ and $F_{\tau}(\cdot)$ denote the probability density function and cumulative distribution function, respectively, of demand if the firm is type $\tau$. We assume that $f_{\tau}$ is greater than 0 over a closed interval on $\Re^{+}$and 0 elsewhere. The demand distribution for a type $\tau_{H}$ firm first order stochastically dominates (FOSD) the demand distribution for a type $\tau_{L}$ firm, i.e., $F_{L}(x) \geq F_{H}(x)$ for all $x \in \Re^{+}$and $F_{L}(x)>F_{H}(x)$ for some $x$.

Orders are placed in multiples of lot size $Q$, i.e., $\eta=n Q$ for some integer $n$. A model in which orders can only be multiples of a fixed quantity commonly occurs in practice, where $Q$ may represent a container, pallet, case pack size or production batch. At one extreme, as $Q$ becomes large, the model captures "all or nothing" investment decisions faced by the firm; at the opposite extreme, the model behaves like an inventory model with a continuous stocking decision. We discuss the implications of the size of $Q$ in Section 5 .

All the parameters in the model except the firm's type are common knowledge to the firm and the equity holder. The firm moves first. At the start of period 1, she receives a private signal 
about her type. Then the firm chooses a stocking quantity $\eta$, which may convey information about her type to the equity holder. The equity holder observes the firm's stocking decision but not her type. He moves second by assigning a short-term valuation or a price to the firm. Subsequently, in period 2, the demand is realized and the firm makes a profit or a loss. The firm is then dissolved and its proceeds are distributed to the equity holder. This time-line is supported by the classical lead time argument in the newsvendor model. ${ }^{1}$

The equity holder's prior beliefs of the firm's type are denoted by $g(\tau)$. His posterior beliefs of the firm's type after seeing the firm's signal $\eta$ are denoted as $\lambda(\tau \mid \eta)$. After receiving signal $\eta$, the equity holder responds by offering a price for the firm, $\rho(\eta) \in P(\eta)$. The set of the equity holder's pure-strategy best responses to signal $\eta$ is represented as $P^{*}\left(T^{\prime}, \eta\right)$, where $\eta$ is the observed stocking quantity and $T^{\prime}$ represents his posterior assessment of firm types, i.e., $T^{\prime}$ is a non-empty subset of $T$ such that $\lambda\left(T^{\prime} \mid \eta\right)=1$.

The firm's utility is a linear combination of the equity holder's valuations of the firm in period 1 and in period 2 with weights $\alpha$ and $1-\alpha$, respectively, where $\alpha \in[0,1]$. A larger value of $\alpha$ corresponds to a higher emphasis on short-term valuation. Note that the valuation of the firm in period 2 would be identical to its actual profit because the firm is liquidated. The firm's expected profit function is directly from the newsvendor model, $\pi(\tau, \eta)=E_{\tau}\left[r \min \{\eta, x\}+s(\eta-x)^{+}-c \eta\right]$. Therefore, the firm's expected utility function is given by

$$
U_{N}(\tau, \eta, \rho(\eta))=\alpha \rho(\eta)+(1-\alpha) \pi(\tau, \eta)
$$

The equity holder operates in a perfectly competitive market and seeks to maximize his expected utility, which depends on his valuation error of the firm. To capture this, we adopt a utility function for the equity holder suggested in Gibbons (1992) that is of the form

$$
U_{E}(\tau, \eta, \rho(\eta))=-[\pi(\tau, \eta)-\rho(\eta)]^{2} .
$$

Instead of assuming a single equity holder with this utility function, we could have assumed that the firm's equity is traded in an efficient market comprised of many investors, which then determines the valuation. This alternative would lead to the same pricing function as the above utility function does, and thus, has no bearing on the results. Assuming a single equity holder enables us to model the actions of the equity holder more clearly.

\footnotetext{
${ }^{1}$ We thank an anonymous reviewer for this observation.
} 


\section{Signaling Game Analysis}

In this section, we develop the conditions that support different equilibria. These conditions are then applied in the numerical analysis in Section 5. We focus our analysis on situations in which neither the firm nor the equity holder pursues dominated strategies or makes mistakes in solving the respective utility maximization problems. In addition, we consider only pure strategies by the players.

\subsection{Complete Information}

Under complete information, the firm's utility function in (1) simplifies to the newsvendor expected profit function. We use $\eta_{L}^{*}$ to denote the smallest order quantity that maximizes the utility of a type $\tau_{L}$ firm when $\lambda\left(\tau_{L} \mid \eta\right)=1$, and $\eta_{H}^{*}$ to denote the smallest order quantity that maximizes the utility of a type $\tau_{H}$ firm when $\lambda\left(\tau_{H} \mid \eta\right)=1$. There can be alternative optimal solutions because $\eta$ is discrete. We use the minimum over the alternative optimal solutions in order to make the exposition unambiguous, but the primary results of our analysis are the same if we instead used the alternative maximizers.

$$
\eta_{j}^{*}=\min \left\{\eta: \underset{\eta}{\arg \max } U_{N}\left(\tau_{j}, \eta, \rho\left(\eta \mid \lambda\left(\tau_{j} \mid \eta\right)=1\right)\right)\right\}, \quad j=L, H .
$$

The classical newsvendor result is also recovered when the firm's short-termism $(\alpha)$ is equal to zero. In this case, the firm's utility function is determined solely by its expected long-term valuation, which the firm again optimizes by a straight application of the newsvendor model.

While the classical newsvendor result is recovered when there is no information asymmetry or no short-termism, the motivation for the firm is different in the two cases. In the former, both players in the game have the same information, so there is nothing to be gained if the firm were to act in a way that was not in accordance with its type, even if the firm had an interest in its short-term valuation. In the latter case, regardless of whether there is information asymmetry, the firm has no interest in its short-term valuation and is motivated solely to optimize its long term valuation. Both information asymmetry and short-termism must be present in order for the firm to deviate from its long-term optimal stocking quantity. 


\subsection{Incomplete Information}

In our analysis under incomplete information, we provide an existence proof for a pooling PBE (Proposition 1 and Corollary 1), show the conditions under which a separating equilibrium does not exist (Proposition 2), show the conditions under which the pooling PBE identified in Proposition 1 survives the Intuitive Criterion refinement (Proposition 3), show the conditions under which a pooling PBE survives the Undefeated refinement (Proposition 4), and identify that in the case of multiple PBE there will be a unique PBE which is a LMSE. According to Kreps and Sobel (1992), a pooling equilibrium is a $\mathrm{PBE}$ in which the firm chooses the same strategy regardless of its type, and a separating equilibrium is a PBE in which each type of firm chooses a different strategy.

We use the following definition of a PBE derived from Fudenberg and Tirole (1991), restated in our notation and reflecting our focus on pure strategies.

Definition 1. Perfect Bayesian Equilibrium. A PBE of a signaling game consists of a strategy profile, $\sigma^{*}$, and posterior beliefs, $\lambda(\tau \mid \eta)$. In the context of pure strategies, a strategy profile for the firm (player $\mathrm{N}$ ), $\sigma_{N}(\tau)$, is an order quantity, $\eta$, for each firm type, $\tau$. A strategy profile for the equity holder (player E), $\sigma_{E}(\eta)$, is an equity price, $\rho(\eta)$, assigned to the firm for each order quantity of the firm, $\eta$. The strategy profiles must be such that for the firm, $\sigma_{N}^{*}(\tau)=\arg \max _{\eta} U_{N}(\tau, \eta, \rho(\eta))$, for all $\tau$. For the equity holder, $\sigma_{E}^{*}(\eta)=\arg \max _{\rho} \sum_{\tau} \lambda(\tau \mid \eta) U_{E}(\tau, \eta, \rho(\eta))$, for all $\eta$.

In addition, if $\sum_{\tau^{\prime} \in T} g\left(\tau^{\prime}\right) \mathbf{1}\left[\sigma_{N}^{*}\left(\tau^{\prime}\right)=\eta\right]>0$ so that Bayes rule can be applied (where $\mathbf{1}[\cdot]$ is an indicator function), the equity holder's posterior beliefs are $\lambda(\tau \mid \eta)=\frac{g(\tau) \mathbf{1}\left[\sigma_{N}^{*}(\tau)=\eta\right]}{\sum_{\tau^{\prime} \in T} g\left(\tau^{\prime}\right) \mathbf{1}\left[\sigma_{N}^{*}\left(\tau^{\prime}\right)=\eta\right]}$. If $\sum_{\tau^{\prime} \in T} g\left(\tau^{\prime}\right) \mathbf{1}\left[\sigma_{N}^{*}\left(\tau^{\prime}\right)=\eta\right]=0$, then Bayes rule cannot be applied and the equity holder's posterior beliefs, $\lambda(\tau \mid \eta)$, may be any probability distribution on $T$.

Intuitively, in a PBE no player has an incentive to deviate from the equilibrium. The equity holder optimizes his utility by setting a price that reflects his posterior beliefs, which are formed after observing the firm's choice of stocking quantity. The firm chooses a stocking quantity while recognizing that this choice may lead the equity holder to update his beliefs. 
The equity holder's best response conditional on his posterior beliefs and the stocking quantity is given by:

$$
\begin{aligned}
\rho^{*}(\eta) & =\underset{\rho}{\arg \max } \sum_{\tau} \lambda(\tau \mid \eta) U_{E}(\tau, \eta, \rho(\eta)) \\
& =\underset{\rho}{\arg \max }\left[-\lambda\left(\tau_{L} \mid \eta\right)\left\{\pi\left(\tau_{L}, \eta\right)-\rho(\eta)\right\}^{2}-\lambda\left(\tau_{H} \mid \eta\right)\left\{\pi\left(\tau_{H}, \eta\right)-\rho(\eta)\right\}^{2}\right]
\end{aligned}
$$

This gives the price assigned by the equity holder as:

$$
\rho^{*}(\eta)=\lambda\left(\tau_{L} \mid \eta\right) \pi\left(\tau_{L}, \eta\right)+\lambda\left(\tau_{H} \mid \eta\right) \pi\left(\tau_{H}, \eta\right)
$$

The resulting price function is a weighted average of the expected profits for each firm type based on the equity holder's posterior belief that the firm is in fact that type. Note that the price is a function of both $\eta$ and $\lambda(\cdot)$. We write the price function as $\rho^{*}(\eta)$ when the posterior beliefs are clear from the context, and as $\rho(\eta \mid \lambda(\tau \mid \eta))$ when we refer to the price for a specific posterior belief.

With this price function, the firm's utility in (1) can be rewritten as

$$
U_{N}\left(\tau, \eta, \rho^{*}(\eta)\right)= \begin{cases}\left(1-\alpha+\alpha \lambda\left(\tau_{L} \mid \eta\right)\right) \pi\left(\tau_{L}, \eta\right)+\alpha \lambda\left(\tau_{H} \mid \eta\right) \pi\left(\tau_{H}, \eta\right) & \text { for } \tau=\tau_{L}, \\ \alpha \lambda\left(\tau_{L} \mid \eta\right) \pi\left(\tau_{L}, \eta\right)+\left(1-\alpha+\alpha \lambda\left(\tau_{H} \mid \eta\right)\right) \pi\left(\tau_{H}, \eta\right) & \text { for } \tau=\tau_{H} .\end{cases}
$$

It is useful to distinguish among three specific valuations of the firm by the equity holder that lead to different order quantities. A low valuation occurs when the equity holder sets the posterior beliefs as $\lambda\left(\tau_{L} \mid \eta\right)=1$, a weighted valuation occurs when the equity holder sets the posterior beliefs as $\lambda(\tau \mid \eta)=g(\tau)$ so they are equal to the prior beliefs, and a high valuation corresponds to $\lambda\left(\tau_{H} \mid \eta\right)=1$. First order stochastic dominance implies that $U_{N}\left(\tau, \eta, \rho^{*}(\eta)\right)$ and the optimal order quantity for a given type $\tau$ are both non-decreasing in $\lambda\left(\tau_{H} \mid \eta\right)$.

Now consider the posterior beliefs of the equity holder. The definition of a PBE defines strategy profiles of the players, but does not fully characterize the posterior beliefs. According to Definition 1, the posterior beliefs at equilibrium points are given by Bayes Rule. For example, if there exists a pooling equilibrium in which the firm stocks $\hat{\eta}$ regardless of its type, then the posterior beliefs of the equity holder in equilibrium will be equal to his prior beliefs, i.e., $\lambda(\tau \mid \hat{\eta})=g(\tau)$. However, Bayes rule cannot be applied on out-of-equilibrium (OOE) paths because $\sum_{\tau^{\prime} \in T} g\left(\tau^{\prime}\right) \mathbf{1}\left[\sigma_{N}^{*}\left(\tau^{\prime}\right)=\eta\right]=0$ for all other choices of $\eta$.

The equity holder could, in theory, have any arbitrary OOE beliefs about the type of the newsvendor. The literature suggests many refinements of varying restrictiveness to determine OOE 
beliefs that are reasonable and any resulting equilibrium is hence justifiable. We apply strict dominance, which is a mild requirement that eliminates those signals for the first player that are strictly dominated with respect to all possible responses from the second player. In sections 4.2.1 and 4.2.2 we go on to apply the more restrictive Intuitive Criterion and Undefeated refinements.

Definition 2. Strict Dominance. Mas-Colell et al. (1995, p.469) state that a signal, $\eta$, is strictly dominated for a type $\tau_{i} \in T$ if there exists another signal $\eta^{\prime}$ such that the following inequality holds:

$$
\max _{\rho(\eta) \in P^{*}(T, \eta)} U_{N}\left(\tau_{i}, \eta, \rho^{*}(\eta)\right)<\min _{\rho\left(\eta^{\prime}\right) \in P^{*}\left(T, \eta^{\prime}\right)} U_{N}\left(\tau_{i}, \eta^{\prime}, \rho^{*}\left(\eta^{\prime}\right)\right) .
$$

Form the set $S(\eta)$ consisting of all types $\tau_{i}$ such that this inequality does not hold. Then a PBE has reasonable beliefs if for all $\eta$ with $S(\eta) \neq \emptyset, \lambda\left(\tau_{i} \mid \eta\right)>0$ only if $\tau_{i} \in S(\eta)$.

In words, equation (5) states that a signal is strictly dominated for a firm type if the best utility which that type could possibly achieve by sending that signal is strictly lower than the worst utility which that type could possibly achieve by sending some other signal. A PBE has reasonable beliefs if those beliefs do not put a positive probability on any type sending a signal that is strictly dominated. Applying strict dominance, we find that there exists a threshold order quantity, $\eta^{s}$, such that the equity holder will be certain that the newsvendor is of type $\tau_{H}$ if and only if he observes an order quantity equal to or greater than $\eta^{s}$. This result is stated in the following lemma. All proofs are in the Appendix.

Lemma 1. There exists an order quantity $\eta^{s}$ defined as

$$
\eta^{s}=\min \left\{\eta: \eta \geq \eta_{L}^{*} \& U_{N}\left(\tau_{L}, \eta, \rho\left(\eta \mid \lambda\left(\tau_{H} \mid \eta\right)=1\right)\right)<U_{N}\left(\tau_{L}, \eta_{L}^{*}, \rho\left(\eta_{L}^{*} \mid \lambda\left(\tau_{L} \mid \eta_{L}^{*}\right)=1\right)\right)\right\}
$$

such that the equity holder's posterior belief will be $\lambda\left(\tau_{H} \mid \eta\right)=1$ if $\eta \geq \eta^{s}$ and $\lambda\left(\tau_{H} \mid \eta\right)<1$ otherwise.

Intuitively, $\eta^{s}$ is the smallest order quantity such that a type $\tau_{L}$ firm receives a lower utility by stocking at or above $\eta^{s}$ under a high valuation than by stocking $\eta_{L}^{*}$ under a low valuation. It represents the smallest separating order quantity because the firm must stock at or above this quantity in order to be considered of type $\tau_{H}$ and receive the corresponding valuation. As a technical note to Lemma 1, in some circumstances $\eta>\eta^{s}$ is dominated for all firm types. No firm type has an incentive to send such a signal nor can they credibly threaten to send such a signal. We limit our analysis to situations in which the firm does not make mistakes or pursue dominated strategies. Thus, only order quantities between $\eta_{L}^{*}$ and $\eta^{s}$ need to be considered. 
Let $\eta^{p}$ be the smallest order quantity that maximizes the expected utility of the type $\tau_{H}$ newsvendor under weighted valuation, i.e.,

$$
\eta^{p}=\min \left\{\eta: \underset{\eta}{\arg \max } U_{N}\left(\tau_{H}, \eta, \rho(\eta \mid \lambda(\tau \mid \eta)=g(\tau))\right)\right\} .
$$

Having specified the price function of the equity holder, the utility function of the newsvendor, and OOE beliefs that are strictly dominated, we now prove the existence of the following pooling equilibrium at $\eta^{p}$.

Proposition 1. If the following three conditions hold:

$$
\begin{aligned}
\eta_{H}^{*} & <\eta^{s}, \\
U_{N}\left(\tau_{L}, \eta^{p}, \rho^{*}\left(\eta^{p}\right)\right) & >U_{N}\left(\tau_{L}, \eta_{L}^{*}, \rho^{*}\left(\eta_{L}^{*}\right)\right), \\
U_{N}\left(\tau_{H}, \eta^{p}, \rho^{*}\left(\eta^{p}\right)\right) & >U_{N}\left(\tau_{H}, \eta^{s}, \rho^{*}\left(\eta^{s}\right)\right),
\end{aligned}
$$

then $\eta^{p}<\eta^{s}$ and there exists a PBE in which the firm stocks $\eta^{p}$ regardless of its type, the equity holder's response function $\rho^{*}(\eta)$ is given by (3), and the equity holder's posterior beliefs are given by

$$
\lambda\left(\tau_{L} \mid \eta\right)=1-\lambda\left(\tau_{H} \mid \eta\right) ; \quad \lambda\left(\tau_{H} \mid \eta\right)= \begin{cases}0 & \eta<\eta^{p} \\ g\left(\tau_{H}\right) & \eta^{p} \leq \eta<\eta^{s} \\ 1 & \eta \geq \eta^{s}\end{cases}
$$

Inequalities (7), (8), and (9) are independent of one another and imply different requirements. Inequality (7) states that the smallest order quantity at which a type $\tau_{H}$ firm can separate from a type $\tau_{L}$ firm must exceed the optimal stocking quantity for a type $\tau_{H}$ firm as determined when there is no information asymmetry between the firm and the equity holder. If this condition is violated then a type $\tau_{H}$ firm will separate by choosing the optimal stocking quantity for her type, $\eta_{H}^{*}$, as doing so would maximize her expected utility and the type $\tau_{L}$ firm would not follow.

Inequality (8) states that the utility derived by a type $\tau_{L}$ firm from stocking $\eta^{p}$ must be larger than the utility derived by a type $\tau_{L}$ firm stocking $\eta_{L}^{*}$. Inequality (9) states that the utility derived by a type $\tau_{H}$ firm from stocking $\eta^{p}$ must be larger than the utility derived by a type $\tau_{H}$ firm stocking $\eta^{s}$, which represents the lowest separating stocking quantity when (7) holds. Note that as $Q$ becomes smaller and the choice set for $\eta$ becomes larger, then (8) will be violated only if (9) is violated. Example 1 in Section 5.1 shows a pooling PBE at $\eta^{p}$. 
Other pooling PBE may exist using a different reasonable belief structure for the equity holder. For example, let $\eta^{g p}$ be any order quantity less than $\eta^{s}$, then Corollary 1 (proof omitted) specifies all possible values of $\eta^{g p}$ at which there will be a pooling PBE under reasonable beliefs. We use the results of this corollary in the application of the Undefeated refinement in section 4.2.2.

Corollary 1. If the following three conditions hold:

$$
\begin{aligned}
\eta_{H}^{*} & <\eta^{s}, \\
U_{N}\left(\tau_{L}, \eta^{g p}, \rho^{*}\left(\eta^{g p}\right)\right) & >U_{N}\left(\tau_{L}, \eta_{L}^{*}, \rho^{*}\left(\eta_{L}^{*}\right)\right), \\
U_{N}\left(\tau_{H}, \eta^{g p}, \rho^{*}\left(\eta^{g p}\right)\right) & >U_{N}\left(\tau_{H}, \eta^{s}, \rho^{*}\left(\eta^{s}\right)\right),
\end{aligned}
$$

then there exists a pooling PBE in which the firm stocks $\eta^{g p}<\eta^{s}$ regardless of its type, the equity holder's response function $\rho^{*}(\eta)$ is given by (3), and posterior beliefs which are reasonable under strict dominance are given by

$$
\lambda\left(\tau_{L} \mid \eta\right)=1-\lambda\left(\tau_{H} \mid \eta\right) ; \quad \lambda\left(\tau_{H} \mid \eta\right)= \begin{cases}0 & \eta<\eta^{g p} \\ g\left(\tau_{H}\right) & \eta=\eta^{g p} \\ 0 & \eta^{g p}<\eta<\eta^{s} \\ 1 & \eta \geq \eta^{s} .\end{cases}
$$

Corollary 1 identifies all possible pooling PBE under reasonable beliefs since (11) represents the posterior beliefs that are most conducive to a pooling PBE under strict dominance. Other posterior beliefs may also support these pooling PBE. Example 1 in Section 5.1 shows multiple pooling PBE identified using Corollary 1.

The literature has focused on the least cost separating PBE as the unique PBE in the case of continuous stocking quantities. In order to be consistent with and to contrast our results against this literature, we now consider separating PBE. In our context, the least cost separating PBE has the $\tau_{L}$ firm choosing $\eta=\eta_{L}^{*}$ and the $\tau_{H}$ firm choosing $\eta=\max \left(\eta^{s}, \eta_{H}^{*}\right)$, which represents the type $\tau_{H}$ firm's optimal stocking quantity choice in a separating PBE under reasonable beliefs. We show in Proposition 2 that with discrete stocking quantities, there are conditions under which a separating PBE does not exist. In section 4.2.1, we show that even when a separating PBE exists, it may not be the unique PBE to survive the Intuitive Criterion refinement (i.e., one or more pooling PBE may also survive this refinement when stocking quantities are discrete). In section 4.2.2, we 
show that when a pooling PBE exists under Corollary 1, no separating PBE using reasonable beliefs will survive the Undefeated refinement. However, when a pooling PBE does not exist, the least cost separating PBE will be the unique PBE to survive this refinement.

Proposition 2. If $\eta_{H}^{*} \geq \eta^{s}$, then the least cost separating PBE will exist under any reasonable belief structure. If $\eta_{H}^{*}<\eta^{s}$, then the least cost separating PBE cannot exist under any reasonable belief structure unless:

$$
U_{N}\left(\tau_{H}, \eta^{s}, \rho^{*}\left(\eta^{s}\right)\right) \geq \max _{\eta^{\prime} \neq \eta^{s}} U_{N}\left(\tau_{H}, \eta^{\prime}, \rho\left(\eta^{\prime} \mid \lambda\left(\tau_{L} \mid \eta^{\prime}\right)=1\right)\right)
$$

Intuitively, if $\eta_{H}^{*} \geq \eta^{s}$, then the type $\tau_{H}$ firm will always separate as this will maximize both her short-term valuation and long-term expected profits. If $\eta_{H}^{*}<\eta^{s}$ and (12) does not hold, then the maximum utility that a type $\tau_{H}$ firm can achieve by separating is strictly less than the utility that she could achieve by choosing the optimal stocking quantity under the low valuation. The type $\tau_{H}$ firm's stocking quantity in the least cost separating PBE is therefore strictly dominated by the type $\tau_{H}$ firm's optimal stocking quantity under the low valuation. Example 2 in Section 5.1 provides an instance of when a separating PBE does not exist.

Note that, if (12) holds, then there will be a separating PBE under some reasonable belief structure, namely $\lambda\left(\tau_{H} \mid \eta\right)=1$ for stocking quantity $\eta \geq \eta^{s}$ and $\lambda\left(\tau_{H} \mid \eta\right)=0$ for $\eta<\eta^{s}$. If a separating PBE does not exist under Proposition 2, then there will be a reasonable belief structure under which a pooling PBE under Corollary 1 will exist. Thus, the conditions in Corollary 1 and Proposition 2 cover all possibilities, but are not mutually exclusive or disjoint. Both the least cost separating PBE and potentially multiple pooling PBEs may exist for the same scenario but utilizing different out-of-equilibrium beliefs. The resulting multiplicity of equilibria motivates the discussion on refinements in Sections 4.2.1 and 4.2.2.

\subsubsection{The Intuitive Criterion Refinement}

We consider whether the pooling PBE stated in Proposition 1 will survive the Intuitive Criterion refinement. The least cost separating PBE survives the Intuitive Criterion refinement by construction. This refinement is applied by evaluating all possible out-of-equilibrium stocking quantities for a particular PBE and identifying whether, compared to the PBE results, a stocking quantity 
exists which would not provide a type $\tau_{L}$ firm with a higher utility using a high valuation but would provide a type $\tau_{H}$ firm with a higher utility using a high valuation. If such a stocking quantity does exist then the PBE is eliminated. The formal definition of the Intuitive Criterion refinement is developed in Cho and Kreps (1987) and provided in the Appendix using our notation.

The following proposition gives the conditions for the pooling PBE at $\eta^{p}$ to survive the Intuitive Criterion.

Proposition 3. The pooling PBE identified in Proposition 1 will survive the Intuitive Criterion refinement if and only if there does not exist a stocking quantity, $\eta^{\prime}$, for which both of the following conditions are true:

$$
\begin{aligned}
& U_{N}\left(\tau_{L}, \eta^{p}, \rho^{*}\left(\eta^{p}\right)\right)>U_{N}\left(\tau_{L}, \eta^{\prime}, \rho\left(\eta^{\prime} \mid \lambda\left(\tau_{H} \mid \eta^{\prime}\right)=1\right)\right), \\
& U_{N}\left(\tau_{H}, \eta^{p}, \rho^{*}\left(\eta^{p}\right)\right)<U_{N}\left(\tau_{H}, \eta^{\prime}, \rho\left(\eta^{\prime} \mid \lambda\left(\tau_{H} \mid \eta^{\prime}\right)=1\right)\right) .
\end{aligned}
$$

In words, the first condition states that the utility for the type $\tau_{L}$ firm is greater at the pooling PBE involving $\eta^{p}$ than at an alternative stocking quantity, $\eta^{\prime}$, under a high valuation. The second condition states that the utility for the type $\tau_{H}$ firm is less at the pooling PBE involving $\eta^{p}$ than at this alternative stocking quantity, $\eta^{\prime}$, under a high valuation. If an alternative stocking quantity, $\eta^{\prime}$, which meets both conditions does not exist then the equilibrium will survive the Intuitive Criterion refinement. Note that these conditions are always satisfied (i.e. an $\eta^{\prime}$ will always exist) when $Q$ is sufficiently small. Therefore, no pooling PBE will survive the Intuitive Criterion refinement if the decision space is continuous.

By replacing $\eta^{p}$ with $\eta^{g p}$, Proposition 3 can equivalently be used to test whether any of the pooling PBE identified by Corollary 1 also survive the Intuitive Criterion refinement. Example 1 in Section 5.1 shows an instance in which the pooling PBE at $\eta^{p}$ survives the Intuitive Criterion refinement.

As we show in Section 5, there are several instances in which a pooling PBE at $\eta^{p}$ survives the Intuitive Criterion refinement. Although we include the refinement in our analysis to be conservative, in doing so we may be eliminating otherwise valid pooling PBE in some cases. The Intuitive Criterion refinement is not without controversy. As noted by Bolton and Dewatripont (2005), "as plausible as the Cho-Kreps intuitive criterion may be, it does seem to predict implausible outcomes 
in some situations." Indeed, the application of certain belief-based refinements such as the Intuitive Criterion is unsettled in the game theory literature (Mailath et al. 1993, Riley 2001).

One concern raised in the literature regarding the Intuitive Criterion refinement is that a type $\tau_{H}$ firm is presumed to choose the separating stocking quantity, $\eta^{s}$, even if such a choice is Paretodominated by the pooling stocking quantity, $\eta^{p}$. This is true even as the probability of the firm being a type $\tau_{L}$ approaches zero (Kreps and Sobel 1992, Bolton and Dewatripont 2005). This results in a discontinuity in the choice of stocking quantity for a type $\tau_{H}$ firm (from $\eta^{s}$ to $\eta_{H}^{*}$ ) when $g\left(\tau_{L}\right)$ goes from a value of $\epsilon>0$ to 0 (Mailath et al. 1993). Example 3 in Section 5.1 illustrates this criticism.

A second criticism is that the Intuitive Criterion assumes that the participants in the game can communicate counterfactual information to other participants by way of "speeches," but these speeches are not explicitly modeled in the game (Salanie 2005). One implication of this is that the equity holder's beliefs, specifically their beliefs at the proposed out-of-equilibrium point, are not fully updated by the application of the Intuitive Criterion. This may cast doubt on whether the deviation proposed by the Intuitive Criterion can actually be considered an unambiguous signal of the firm's type (Mailath et al. 1993).

\subsubsection{The Undefeated Refinement}

In light of the criticisms that have been raised concerning the Intuitive Criterion refinement and since in many cases it fails to identify a unique PBE, we extend our analysis by considering whether the pooling PBE stated in Proposition 1 will survive the Undefeated refinement. The Undefeated refinement is applied by iterating across all of the possible PBE in the model and identifying whether the beliefs used to support each PBE are reasonable given the other possible PBE and the preferences for each firm type among those PBE. PBE that rely on beliefs that are unreasonable in this regard are eliminated. The formal definition of the Undefeated refinement is developed in Mailath et al. (1993) and provided in the Appendix using our notation.

The Undefeated refinement has been applied in the finance and economics literature (Spiegel and Spulber 1997, Taylor 1999, Gomes 2000, Fishman and Hagerty 2003) and it addresses many of the concerns raised about the Intuitive Criterion refinement. By construction the Undefeated refinement does not eliminate any PBE that is Pareto efficient, as is possible with the Intuitive Cri- 
terion refinement. In addition, unlike the Intuitive Criterion refinement, the Undefeated refinement does not rely on unmodeled "speeches" from the firm in order to convey additional information to the equity holder. Instead, the Undefeated refinement ensures that out-of-equilibrium beliefs are restricted only by other equilibria in the model.

Proposition 4. If one or more pooling PBE exists under reasonable beliefs as in Corollary 1, then (i) at least one of those PBE will survive the Undefeated refinement, and (ii) the least cost separating PBE, if it exists, will not survive.

The intuition behind Proposition 4 is that at least one of the pooling PBE identified using Corollary 1 will not be Pareto dominated by any other PBE. If the least cost separating equilibrium also exists under Proposition 2 then every pooling PBE that exists under Corollary 1 is by definition a Pareto improvement over the separating PBE. A corollary result to Proposition 4 is that if a pooling PBE does not exist under reasonable beliefs then the least cost separating PBE is the unique Undefeated PBE. Example 1 in Section 5.1 illustrates these possibilities.

When multiple pooling PBE exist under reasonable beliefs, the Undefeated refinement may identify a unique PBE. However, it may also be that more than one pooling PBE survives the Undefeated refinement. If multiple pooling PBE survive the Undefeated refinement and a unique PBE is desired, an additional constraint must be added to the model. Mailath et al. (1993) utilizes the concept of lexicographically maximum sequential equilibrium (LMSE) to identify a unique PBE when multiple PBE survive the Undefeated refinement. A PBE is a LMSE if among all PBE it maximizes the utility for the type $\tau_{H}$ firm and conditional on maximizing the utility for the type $\tau_{H}$ firm, it then maximizes the utility for the type $\tau_{L}$ firm. Using a LMSE to identify a unique PBE is intuitively appealing because typically a low-quality firm wishes to masquerade as a high-quality firm rather than the opposite, so resolving on a belief structure that supports such an outcome seems reasonable (Taylor 1999). The alternative would be to use a belief structure that increases the utility of a type $\tau_{L}$ firm but decreases the utility of a type $\tau_{H}$ firm compared to the LMSE, which is more difficult to justify.

Due to the concavity of the utility functions, a unique LMSE will always exist among the PBE that survive the Undefeated refinement. If one of the pooling PBEs is at $\eta^{p}$, then this will be the unique PBE which is a LMSE since it maximizes the utility of a type $\tau_{H}$ firm and conditional on that, maximizes the utility of a type $\tau_{L}$ firm. 


\section{Numerical Analysis}

The objective of the numerical analysis is to evaluate the effect of the model parameters on the existence of a pooling PBE and whether it will survive the Intuitive Criterion and Undefeated refinements. We present specific examples in Section 5.1 and observations from an exhaustive computational study in Section 5.2.

\subsection{Illustrative Examples}

Suppose that demand follows exponential distributions with means $\mu_{L}=500$ and $\mu_{H}=800$, the price parameters are $p=1.00, c=0.60, s=0.00$, the extent of short-termism is $\alpha=0.80$, and the probability that the firm is type $\tau_{L}$ is $g\left(\tau_{L}\right)=0.20$.

Example 1. Let $Q=50$. We find that $\eta_{L}^{*}=250, \eta_{H}^{*}=\eta^{p}=400$, and $\eta^{s}=650$. Stocking quantity $\eta_{H}^{*}$ happens to be equal to $\eta^{p}$, but this need not be the case in general. Importantly, however, $\eta^{s}$ is greater than $\eta_{H}^{*}$. Figure 1a displays the utility functions for a $\tau_{L}$ type firm under the low, weighted and high valuations, and for a $\tau_{H}$ type firm under the weighted and high valuations, with the solid points representing the achievable utilities for each firm type given that the stocking quantity is a multiple of $Q$. Points A and $\mathrm{E}$ in the figure show that stocking equal to or greater than $\eta^{s}$ will provide a type $\tau_{L}$ firm with a lower expected utility under a high valuation than stocking $\eta_{L}^{*}=250$ under a low valuation. Under the definition of strict dominance, reasonable beliefs by the equity holder should place zero probability that a firm stocking $\eta \geq \eta^{s}$ is a type $\tau_{L}$ firm.

We apply the three conditions of Proposition 1. The relevant expected utilities for this are $U_{N}\left(\tau_{L}, \eta^{p}, \rho^{*}\left(\eta^{p}\right)\right)=60.6, U_{N}\left(\tau_{L}, \eta_{L}^{*}, \rho^{*}\left(\eta_{L}^{*}\right)\right)=46.7, U_{N}\left(\tau_{H}, \eta^{p}, \rho^{*}\left(\eta^{p}\right)\right)=68.5$ and $U_{N}\left(\tau_{H}, \eta^{s}, \rho^{*}\left(\eta^{s}\right)\right)=$ 55.0 , and $\eta^{s}$ is greater than $\eta_{H}^{*}$. Thus, all three conditions are met and a pooling PBE exists at $\eta^{p}=$ 400. The expected utilities are shown in Figure 1a by points labeled A, B, C and D, respectively.

Using Corollary 1, all of the pooling PBE can be identified in a similar fashion to be at $\eta=$ 200, 250, 300, 350, 400, 450, and 500. The least cost separating PBE also exists in which the type $\tau_{H}$ firm stocks $\eta^{s}=650$ and the type $\tau_{L}$ firm stocks $\eta_{L}^{*}=250$. This PBE survives the Intuitive Criterion refinement by construction. Based on Proposition 3, the pooling PBEs at 400 and 500 also survive the Intuitive Criterion refinement. Figure $1 \mathrm{~b}$ shows this in greater detail for the pooling PBE at $\eta^{p}=400$. Compared to the pooling PBE at $\eta^{p}=400$, the $\tau_{L}$ firm is willing to stock up to 500 units in order to receive a high valuation, but the type $\tau_{H}$ firm is unwilling to stock more 
than 500 units in order to receive a high valuation in lieu of the weighted valuation. Therefore, as required by Proposition 3, there is no stocking quantity to which a type $\tau_{H}$ firm is willing to deviate under a high valuation but a type $\tau_{L}$ firm is unwilling to deviate under a high valuation.

Of all the possible pooling and separating PBE, the pooling PBEs at $\eta=350$ and 400 survive the Undefeated refinement but the least cost separating PBE does not, in accordance with Proposition 4. The pooling PBE at $\eta=350$ defeats the pooling PBE at $\eta=200,250,300,450$, and 500 as well as the separating $\mathrm{PBE}$, while the pooling $\mathrm{PBE}$ at $\eta=400$ defeats the pooling $\mathrm{PBE}$ at $\eta=200$, 250, 450 and 500 as well as the separating PBE. No PBE defeats the pooling PBEs at $\eta=350$ or 400. The pooling PBE $\eta=400$ is the unique PBE which is a LMSE.

Thus, this example shows all the pooling PBE and the least cost separating PBE. It shows that the separating PBE and some of the pooling PBE survive the Intuitive Criterion refinement under Proposition 3, and that only some of the pooling PBE survive the Undefeated refinement under Proposition 4.

Example 2. In this example, a separating PBE does not exist. Let $Q=100$ and the other parameters be the same as in Example 1. We have $\eta_{L}^{*}=300, \eta_{H}^{*}=\eta^{p}=400$, and $\eta^{s}=650$. The type $\tau_{H}$ firm obtains an expected utility of 46.5 by stocking $\eta^{s}$ under high valuation. Under low valuation, its expected utility is maximized by stocking 300 and is equal to 50.5. Thus, by Proposition 2, the firm will choose not to separate. The utilities are illustrated in Figure 2 by points D and F respectively. Although a separating PBE does not exist in this example, multiple pooling PBE exist under Corollary 1 , namely at $\eta=200,300,400$ and 500. The pooling PBE at $\eta=400$ and 500 survive the Intuitive Criterion refinement. The pooling PBE at $\eta=300$ and 400 survive the Undefeated refinement. The pooling PBE at $\eta=400$ is the only PBE which is a LMSE.

Example 3. We highlight the first criticism of the Intuitive Criterion refinement mentioned in Section 4.2.1, namely that it may identify the least cost separating PBE as the unique surviving PBE even if another PBE is a Pareto improvement over it. Let the demand follow exponential distributions with means $\mu_{L}=500$ and $\mu_{H}=1000$, and the remaining parameters be $p=1.00$, $c=0.20, s=0.00, \alpha=0.95, g\left(\tau_{L}\right)=0.05$, and $Q=1$. There is a pooling PBE at $\eta^{p}=1,571$, which results in expected utilities of 463.1 for a type $\tau_{H}$ firm, 447.4 for a type $\tau_{L}$ firm, and 0 for the equity holder. However, this pooling PBE is eliminated by the application of the Intuitive Criterion refinement. In fact, the only equilibrium that survives the Intuitive Criterion refinement 
is the least cost separating PBE in which a type $\tau_{H}$ firm stocks $\eta^{s}=3,543$ and a type $\tau_{L}$ firm stocks $\eta_{L}^{*}=805$. This separating PBE results in a utility of 262.5 for a type $\tau_{H}$ firm (a decrease of $43 \%$ compared to the pooling PBE), a utility of 239.1 for a type $\tau_{L}$ firm (a decrease of $47 \%$ compared to the pooling PBE) and a utility of 0 for the equity holder (no change compared to the pooling PBE so the equity holder is indifferent between the two equilibria). Although the pooling PBE at $\eta^{p}$ is a Pareto improvement over the least cost separating PBE, the Intuitive Criterion implies that both types will instead choose the least cost separating equilibrium.

Example 4. Although we have focused on pooling PBE at $\eta=\eta^{p}$, there are many instances in which pooling PBE at other values of $\eta$ exist and survive refinement. One counter intuitive result entails a $\tau_{H}$ firm adopting the preferred stocking quantity of a $\tau_{L}$ firm. Let the demand follow exponential distributions with $\mu_{L}=500$ and $\mu_{H}=600$, and the remaining parameters be $p=1.00$, $c=0.80, s=0.00, \alpha=0.65, g\left(\tau_{L}\right)=0.50$, and $Q=50$. There is no pooling PBE at $\eta^{p}=150$ units because (8) in Proposition 1 is violated (the utility of the $\tau_{L}$ firm at $\eta^{p}$ is 10.61 and at $\eta_{L}^{*}$ is 10.63 ), so a type $\tau_{L}$ firm would prefer to separate than to stock $\eta^{p}$. There is no separating PBE either, because (12) in Proposition 2 is violated (the utility of a $\tau_{H}$ firm at $\eta^{s}$ is 10.08 and her maximum utility under low valuation is 11.15 ), so a type $\tau_{H}$ firm is unwilling to separate. Under Corollary 1 , however, there is a pooling PBE at $\eta=100$ (the utility of a $\tau_{H}$ firm is 11.63 and of a $\tau_{L}$ firm is 11.11). In fact, this is the unique PBE and as such it naturally survives the Intuitive Criterion and Undefeated refinements. It is interesting to note that this stocking quantity maximizes the utility of a type $\tau_{L}$ firm under a weighted valuation, but it does not maximize the utility of a type $\tau_{H}$ firm under a weighted valuation. It also maximizes the utility of a type $\tau_{L}$ firm if there were no information asymmetry. This indicates that when information asymmetry does exist, there are situations in which a $\tau_{H}$ firm can benefit by adopting the preferred stocking quantity of the $\tau_{L}$ firm.

\section{$5.2 \quad$ Effects of model parameters}

In this analysis, we model 18,240 combinations of the following inputs:

- The mean demand faced by a type $\tau_{H}$ firm, $\mu_{H}$ : 600, 800, 1000. Both firm types face an exponential demand distribution with density $f_{i}(x)=\mu_{i}^{-1} e^{-k_{i} x}$ for $i \in L, H$. The mean demand of a type $\tau_{L}$ firm is $\mu_{L}=500$.

- The critical fractile, $(p-c) /(p-s): 0.2,0.4,0.6,0.8$ 
- The stocking quantity increment, $Q: 1,10,50,100$

- The firm's interest in its short-term valuation, $\alpha$ : 0.05 to 1.00 in increments of 0.05

- The equity holder's prior beliefs that the firm is type $\tau_{L}, g\left(\tau_{L}\right): 0.05$ to 0.95 in increments of 0.05

We use the requirements and definitions outlined in Proposition 1 to check for the existence of a pooling PBE at $\eta=\eta^{p}$. Using Proposition 1 rather than Corollary 1 is a conservative means of achieving our objective as additional pooling PBE may exist under Corollary 1. However, using Proposition 1 simplifies the exposition of the analysis and, as the results show, it is still allows us achieve our objective. In addition, if a pooling PBE exists under Proposition 1 then it also survives the Undefeated refinement under Proposition 4 and it will be the unique LMSE.

Based on the results of the analysis, the existence of a pooling PBE is not a pathological phenomenon. Across the 18,240 scenarios examined, a pooling PBE under Proposition 1 exists in 7,271 cases $(39.8 \%)$. In all these cases, it also survives the Undefeated refinement and is the unique LMSE, because among all possible PBE, this pooling PBE maximizes the utility of a type $\tau_{H}$ firm. In addition, 2,904 of these pooling PBE survive the Intuitive Criterion refinement (39.9\% of all pooling PBEs at $\left.\eta^{p}\right)$.

Figure $3 \mathrm{a}, 3 \mathrm{~b}$ and $3 \mathrm{c}$ provide examples from the numerical analysis. Each figure shows the equilibrium outcomes across the analyzed range of values for $\alpha$ and $g\left(\tau_{L}\right)$. In Figure 3a, the mean of the exponential demand faced by a type $\tau_{H}$ firm is 1000 , the critical fractile is 0.8 , and the stocking quantity increment is 100 . The corresponding values in Figure $3 \mathrm{~b}$ are $\mu_{H}=800$, critical fractile $=0.4$, and $Q=50$, and in Figure 3c, $\mu_{H}=600$, critical fractile $=0.2$, and $Q=10$. In each figure, dark grey boxes identify scenarios for which a pooling PBE at $\eta^{p}$ exists, survives the Undefeated refinement, is the unique LMSE and survives the Intuitive Criterion refinement. Medium grey boxes identify scenarios for which a pooling PBE at $\eta^{p}$ exists, survives the Undefeated refinement, is the unique LMSE but does not survive the Intuitive Criterion refinement. Light grey boxes identify scenarios for which there is no pooling PBE at $\eta^{p}$. In most of the latter cases, the least cost separating $\mathrm{PBE}$ is the unique PBE, but there is an interesting category of exceptions where Example 4 applies, i.e., there are pooling PBE at stocking quantities other than $\eta^{p}$. 
Through an examination of the results of these scenarios and an interpretation of the requirements identified in Propositions 1, 2, 3 and 4, we identify the model parameters that lead to the existence of a pooling PBE:

1. Higher firm interest in its short-term valuation, $\alpha$ : A higher $\alpha$ reflects a greater willingness on the part of the firm to choose a stocking quantity that will increase its shortterm valuation, even if that stocking quantity is detrimental to its long-term expected profits. For instance, at the extreme value of $\alpha=1$, the long-term expected profits of the firm has no influence on the firm's stocking decision and the firm bases its decision solely on the maximization of the equity holder's short-term valuation. For low levels of $\alpha$ both firm types will choose the stocking quantity which optimizes their second period expected value. For instance, at the extreme value of $\alpha=0$, the short-term value of the firm has no influence on the firm's stocking decision and the classical newsvendor result is obtained.

There are two effects of a higher value of $\alpha$ on the actions of a type $\tau_{H}$ firm. The primary effect is that a type $\tau_{H}$ firm is more willing to overstock if doing so allows it to separate from a type $\tau_{L}$ firm and thereby garner a higher short-term valuation. A secondary effect is based on how a higher $\alpha$ influences the actions of a type $\tau_{L}$ firm. As $\alpha$ increases, a type $\tau_{L}$ firm is also more willing to overstock if such an action garners a higher short-term valuation. This increases the value of $\eta^{s}$, which in turn, makes it more expensive for a type $\tau_{H}$ firm to separate and therefore makes a pooling stocking quantity a more attractive choice to a type $\tau_{H}$ firm. As $\alpha$ gets increasingly large, this secondary effect becomes the dominant effect, resulting in a pooling PBE.

2. Lower prior belief that the firm is type $\tau_{L}, g\left(\tau_{L}\right)$ : As $g\left(\tau_{L}\right)$ decreases, two forces make it more attractive for a type $\tau_{H}$ firm to select a pooling stocking quantity. Consider the pooling stocking quantity, $\eta^{p}$, which maximizes the utility of the type $\tau_{H}$ firm under a weighted valuation. First, the equity holder's price function under information asymmetry approaches his price function for a type $\tau_{H}$ firm under no information asymmetry, so the price the firm receives at $\eta^{p}$ increases (refer to Lemma 2). Second, as $g\left(\tau_{L}\right)$ decreases then $\eta^{p}$ approaches $\eta_{H}^{*}$, which increases the expected long-term profits of a type $\tau_{H}$ firm choosing stocking quantity $\eta^{p}$. Both of these factors improve a type $\tau_{H}$ firm's utility at stocking quantity $\eta^{p}$, making it comparatively less attractive for a type $\tau_{H}$ firm to separate by instead choosing stocking 
quantity $\eta^{s}$.

A type $\tau_{L}$ firm also benefits from the increasing value which the equity holder places on the firm. There is an offsetting secondary effect, however. As $g\left(\tau_{L}\right)$ decreases, $\eta^{p}$ increases, which reduces the expected long-term profits of a type $\tau_{L}$ firm choosing stocking quantity $\eta^{p}$. These two forces offset each other to varying degrees and may result in the violation of Inequality (8) in Proposition 1, particularly when the stocking quantity increment $(Q)$ is larger.

3. Smaller difference in the performance prospects between the two types (lower mean of the exponential demand of a type $\tau_{H}$ firm): A smaller difference in the performance prospects between the two firm types is modeled by having the mean of the exponential demand of a type $\tau_{H}$ firm approach that of a type $\tau_{L}$ firm. Under such a circumstance the incremental value achieved by being recognized as a type $\tau_{H}$ firm is not as large and it is therefore less attractive for a type $\tau_{H}$ firm to choose stocking quantity $\eta^{s}$, the separating stocking quantity, if it entails costly overstocking. As a result, as the difference in the performance prospects between the two types shrinks, it is more likely that a pooling PBE will exist. However, in these instances the consequences of a pooling PBE on the expected profits of either firm type are also lower since the two types already face similar demand.

4. Larger stocking quantity increment, $Q$ : If the stocking quantity increment is large, two factors contribute to making a pooling PBE more likely compared to if the stocking quantity increment is small. First, when the stocking quantity increment is large the value of $\eta_{L}^{*}$ may be much different from the value of $\eta_{L}^{*}$ if the stocking quantity increment were instead small. This lowers the utility that a type $\tau_{L}$ firm would achieve by choosing $\eta_{L}^{*}$ and provides a stronger incentive for a type $\tau_{L}$ firm to select the pooling stocking quantity instead. A secondary effect is that the separating stocking quantity, $\eta^{s}$, will be higher, which in turn provides a stronger incentive for a type $\tau_{H}$ firm to select the pooling stocking quantity.

The stocking quantity increment $(Q)$ plays a critical role in determining whether or not a pooling PBE survives the Intuitive Criterion refinement. The coarser the signaling instrument, the less likely there will be a stocking quantity choice that satisfies the conditions of the Intuitive Criterion. One can think of the Intuitive Criterion as specifying a range of stocking quantities. The low end of the range specifies the value above which a type $\tau_{L}$ firm is unwilling to deviate from the 
pooling equilibrium even if it were to result in a high valuation. The high end of this range specifies the value above which a type $\tau_{H}$ firm is unwilling to deviate from the pooling equilibrium even if it were to result in a high valuation. If, due to the coarseness of the stocking quantity increment, there is no stocking quantity choice within this range that a type $\tau_{H}$ firm can choose, then the pooling equilibrium will survive the Intuitive Criterion refinement.

\section{Conclusions}

Unlike previous research which has found that firms with high quality prospects will consistently overinvest in projects when short-termism is present and investors have incomplete information concerning the quality of the firm's prospects, we find that this will not universally be the case. We find that not only do pooling PBE exist, but these PBE will often survive both the Intuitive Criterion and Undefeated refinements. We show that the conditions for a pooling equilibrium at $\eta^{p}$ can be met when there is higher short-termism $(\alpha)$, lower prior belief that the firm is a type $\tau_{L}$ firm $\left(g\left(\tau_{L}\right)\right)$, a smaller difference in the performance prospects between the two types, and a larger stocking quantity increment $(Q)$. These conditions inform both investors and firms regarding when to expect a pooling equilibrium.

The insight obtained from our model compared to the previous literature is primarily due to differences in the assumptions on the granularity of investment opportunities. For instance, in both Bebchuk and Stole (1993) and Lai et al. (2011b) the firm faces an investment decision with continuous support. In contrast, our paper attempts to reflect some of the difficulties that complicate decision making when investment levels are discrete. We have also applied the Undefeated refinement as an alternative to the Intuitive Criterion refinement. Although we demonstrate that in many cases a pooling PBE will result even if the Intuitive Criterion refinement is used, this result is more frequent under the Undefeated refinement.

The investment phenomena captured by our model are present in a variety of real-world situations. French upscale beauty brand Clarins Group provides an example of how one firm avoided the pressures to make sub-optimal investment decisions. This was accomplished when relatives of deceased founder Jacques Courtin-Clarins took the firm private in the summer of 2008. This decision reduced the firm's emphasis on short-term valuation (reducing $\alpha$ ) and mitigated information asymmetry with its equity holders by moving from public market equity holders to private and 
family equity holders. However, did the choice to go private actually help Clarins avoid making sub-optimal investment decisions? Our model indicates that type $\tau_{H}$ firms are in particular jeopardy of under investing in their operations when the investment opportunity is chunky (a high $Q$ ) and there is a general belief among equity holders that the firm faces dim prospects (a high $g\left(\tau_{L}\right)$ ).

This scenario materialized in late 2008 and 2009 as the global recession deepened. Many analysts were generally pessimistic about sales of high-end beauty products that would be discretionary for many consumers and noted that "luring women to invest in high-end skin-care regimens is challenging when shoppers are cutting back" (Byron 2009). Clarins management, presumably because of private information that would have been difficult to communicate to public market investors, saw considerable opportunity to sell its high-end products by dramatically increasing its presence in the U.S. By going private in 2008, the firm was able to invest substantially in opening "Clarins department-store skin spas" in 2009. According to a Wall Street Journal interview with Chairman Christian Courtin-Clarins, going private allowed Clarins to be "free from short-term shareholder pressure, [and] Clarins is investing in long-term objectives" (Byron 2009).

Firms other than Clarins have also argued that in some circumstances they can face "pressures" that prevent them from making investments that are in their long-term interests. For instance, Worldspan and Sabre Holdings, both travel technology providers, have gone private in an attempt "to shrug off short-term shareholder pressure" (Field 2007). Other companies have tried to get around this problem by keeping control in the hands of a few individuals. This is the approach taken by Maersk, the world's largest container shipping firm. Maersk's management is focused on two goals: (1) control as many as possible of the companies that influence its containers' movement from country to country, and (2) pursue scale - both in the size of the firm's main businesses and in its ships. The Financial Times noted, "The company can be single-minded in pursuit of these objectives because it is largely immune from short-term shareholder pressure. The founding McKinney Moller family holds 55 per cent of the shares and 75 per cent of voting stock, with 49.8 per cent of the shares all held by a single family charitable trust" (Wright 2006).

While we hopefully established some interesting results associated with the sometimes counter intuitive impact of granular stocking decisions, there is much more that can be done in this area, including investigating specific applications of firm under- and over-investing. One example is the analysis of supply chain or operational disruptions. The possibility of an operational disruption 
is inherent in every firm, whether due to incompetent operational decision making or unlucky outcomes of competent decisions. There is a third potential source of disruptions that is revealed by our model, namely competent decision making that purposefully sub-optimizes the expected operational performance of the firm. In this light, a firm may undertake operational decisions while anticipating that the long-term result of such a decision will be suboptimal.

Another area for further study is examining the implications of the model across multiple decision periods. For instance, if there are changes to the parameter space due to internal or external shocks over multiple time periods, firms may oscillate among different equilibria over time, and hence, make different investment decisions even though the firm's expected profit function does not necessarily change. An internal shock could be modeled as a change in $\alpha$. Such a shock may occur due to changes in compensation structure or firm strategy. An external shock could be modeled as a change in $g\left(\tau_{L}\right)$. Such a shock may occur due to information revelation about the firm, such as when a large investor takes a substantial stake in a firm. It may also occur due to macro-economic events such as the dot-com boom and subsequent bust, the onset of a recession, or a competitor filing for bankruptcy. It is interesting to note that the firm's investment decision may change even if its type and the expected profits associated with the different investment decisions remain constant. The result will be seemingly erratic investment behavior in which a firm may swing among strategies of under investing, optimally investing, and over investing. This fluctuation in firm behavior is generally in line with the findings of Aghion and Stein (2008), who identify a pattern of oscillation in a firm's choice of business strategy even in the absence of external shocks.

\section{References}

Aghion, Philippe, Patrick Bolton. 1987. Contracts as a barrier to entry. The American Economic Review $77(3)$ 388-401.

Aghion, Philippe, Jeremy C. Stein. 2008. Growth versus margins: Destabilizing consequences of giving the stock market what it wants. Journal of Finance 63 1025-1058.

Allon, Gad, Achal Bassamboo. 2011. Buying from the babbling retailer? The impact of availability information on customer behavior. Management Science 57(4) 713-726.

Anand, Krishnan S., Manu Goyal. 2009. Strategic information management under leakage in a supply chain. Management Science 55(3) 438-452. 
Barton, Dominic. 2011. Capitalism for the long term. Harvard Business Review (March 2011) 3-9.

Bebchuk, Lucian Arye, Lars A. Stole. 1993. Do short-term objectives lead to under- or overinvestment in long-term projects? The Journal of Finance 48(2) 719-729.

Berle, Adolf Augustus, Gardiner Coit Means. 1932. The modern corporation and private property. Macmillan, New York.

Bolton, Gary E., Elena Katok. 2008. Learning by doing in the newsvendor problem: A laboratory investigation of the role of experience and feedback. Manufacturing and Service Operations Management 10(3) $519-538$.

Bolton, Patrick, M. Dewatripont. 2005. Contract theory. MIT Press, Cambridge, Mass.

Bostian, A.J., Charles A. Holt, Angela M. Smith. 2008. Newsvendor "pull-to-center" effect: Adaptive learning in a laboratory experiment. Manufacturing and Service Operations Management 10(4) 590 608 .

Byron, Ellen. 2009. Clarins puts on its best face in U.S. Wall Street Journal; March 23, 2009; Dow Jones, Inc.

Chen, Ying, Navin Kartik, Joel Sobel. 2008. Selecting cheap-talk equilibria. Econometrica 76(1) 117-136.

Cho, In-Koo, David M. Kreps. 1987. Signaling games and stable equilibria. The Quarterly Journal of Economics 102(2) 179-221.

Cramton, Peter C., Joseph S. Tracy. 1992. Strikes and holdouts in wage bargaining: Theory and data. The American Economic Review 82(1) 100-121.

Debo, Laurens, Senthil Veeraraghavan. 2010. Prices and congestion as signals of quality. Working paper, Chicago Booth School of Business, University of Chicago.

Deshpande, Vinayak, Morris A. Cohen, Karen Donohue. 2003. An empirical study of service differentiation for weapon system service parts. Operations Research 51(4) 518-530.

Field, David. 2007. New owners for Worldspan and Sabre. Airline Business 484.

Fishman, Michael J, Kathleen M Hagerty. 2003. Mandatory versus voluntary disclosure in markets with informed and uninformed customers. Journal of Law, Economics, and Organization 19(1) 45-63.

Fudenberg, Drew, Jean Tirole. 1991. Game theory. MIT Press, Cambridge, Mass.

Gibbons, Robert. 1992. Game theory for applied economists. Princeton University Press, Princeton, N.J.

Gomes, Armando. 2000. Going public without governance: Managerial reputation effects. The Journal of Finance 55(2) 615-646.

Graham, John R., Campbell R. Harvey, Shiva Rajgopal. 2005. The economic implications of corporate financial reporting. Journal of Accounting and Economics 40(1-3) 3-73. 
Grinblatt, Mark, Chuan Yang Hwang. 1989. Signalling and the pricing of new issues. The Journal of Finance 44(2) 393-420.

Grossman, Sanford J. 1981. The informational role of warranties and private disclosure about product quality. Journal of Law and Economics 24(3) 461-483.

Holmstrom, Bengt. 1999. Managerial incentive problems: A dynamic perspective. The Review of Economic Studies 66(1) 169-182.

Islegen, O., Erica L. Plambeck. 2007. Capacity leadership in supply chains with asymmetric demand information and noncontractible capacity. Working Paper, Stanford Graduate School of Business.

Jensen, Michael C. 1986. Agency costs of free cash flow, corporate finance, and takeovers. The American Economic Review 76(2) 323-329.

Jensen, Michael C., William H. Meckling. 1976. Theory of the firm: Managerial behavior, agency costs and ownership structure. Journal of Financial Economics 3(4) 305-360.

Kremer, Mirko, Stefan Minner, Luk N. Van Wassenhove. 2010. Do random errors explain newsvendor behavior? Manufacturing and Service Operations Management 12(4) 673-681.

Kreps, David M., Joel Sobel. 1992. Signalling, vol. 11, chap. 25. North-Holland; Elsevier Science Pub. Co., Amsterdam; New York, N.Y., 850-867.

Lai, Guoming, Laurens Debo, Lin Nan. 2011a. Channel stuffing with short-term interest in market value. Management Science 57(2) 332-346.

Lai, Guoming, Wenqiang Xiao, Jun Yang. 2011b. Supply chain performance under market valuation: An operational approach to restore efficiency. Working Paper Series.

Lai, Richard. 2006. Is inventory's fiscal year end effect caused by sales timing? A test using a natural experiment from Germany. Working paper, Harvard Business School.

Mailath, George J., Masahiro Okuno-Fujiwara, Andrew Postlewaite. 1993. Belief-based refinements in signalling games. Journal of Economic Theory 60(2) 241-276.

Mas-Colell, Andreu, Michael Dennis Whinston, Jerry R. Green. 1995. Microeconomic theory. Oxford University Press, New York.

Milgrom, Paul, John Roberts. 1986. Price and advertising signals of product quality. Journal of Political Economy 94(4) 796-821.

Nahmias, Steven. 2001. Production and operations analysis. 4th ed. The McGraw-Hill/Irwin series. Operations and decision sciences, McGraw-Hill/Irwin, Boston.

Narayanan, M. P. 1985. Managerial incentives for short-term results. The Journal of Finance 40(5) 14691484. 
Rappaport, Alfred. 2004. Beyond quarterly earnings. Wall Street Journal; March 8, 2004; Dow Jones, Inc.

Riley, John G. 2001. Silver signals: Twenty-five years of screening and signaling. Journal of Economic Literature 39(2) pp. 432-478.

Salanie, Bernard. 2005. The economics of contracts : A primer. 2nd ed. MIT Press, Cambridge, Mass.

Schweitzer, Maurice E., Grard P. Cachon. 2000. Decision bias in the newsvendor problem with a known demand distribution: Experimental evidence. Management Science 46(3) 404-420.

Spence, Michael. 1974. Market signaling : Informational transfer in hiring and related screening processes, Harvard economic studies ; v. 143, vol. v. 143. Harvard University Press, Cambridge.

Spiegel, Yossef, Daniel F. Spulber. 1997. Capital structure with countervailing incentives. The RAND Journal of Economics 28(1) pp. 1-24.

Stein, Jeremy C. 1988. Takeover threats and managerial myopia. The Journal of Political Economy 96(1) $61-80$.

Stein, Jeremy C. 1989. Efficient capital markets, inefficient firms: A model of myopic corporate behavior. The Quarterly Journal of Economics 104(4) 655-669.

Stein, Jeremy C. 2003. Agency, Information and Corporate Investment, vol. 21, chap. 2. 1st ed. Elsevier North-Holland, Amsterdam; Boston, 110-163.

Taylor, Curtis R. 1999. Time-on-the-market as a sign of quality. The Review of Economic Studies 66(3) $555-578$.

van Donselaar, Karel H., Vishal Gaur, Tom van Woensel, Rob A. C. M. Broekmeulen, Jan C. Fransoo. 2010. Ordering behavior in retail stores and implications for automated replenishment. Management Science 56(5) $766-784$.

Wright, Robert. 2006. Building the engines of globalisation: The story of shipping giant Maersk. Financial Times; October 2, 2006; The Financial Times Ltd.

\section{Appendix}

\subsection{Definitions}

Definition 3. Intuitive Criterion Refinement. According to (Cho and Kreps 1987), the Intuitive Criterion refinement is applied in two steps to evaluate an equilibrium involving $\eta$ and $\rho^{*}(\eta):$ 
1. Form the set $S\left(\eta^{\prime}\right)$ for all $\eta^{\prime} \neq \eta$ consisting of all types $\tau$ such that

$$
U_{N}\left(\tau, \eta, \rho^{*}(\eta)\right)>\max _{\rho\left(\eta^{\prime}\right) \in P^{*}\left(T, \eta^{\prime}\right)} U_{N}\left(\tau, \eta^{\prime}, \rho\left(\eta^{\prime}\right)\right)
$$

2. If, for some out of equilibrium signal $\eta^{\prime}$, there exists some type $\tau^{\prime} \in T \backslash S\left(\eta^{\prime}\right)$ such that

$$
U_{N}\left(\tau^{\prime}, \eta, \rho^{*}(\eta)\right)<\min _{\rho\left(\eta^{\prime}\right) \in P^{*}\left(T \backslash S\left(\eta^{\prime}\right), \eta^{\prime}\right)} U_{N}\left(\tau^{\prime}, \eta^{\prime}, \rho\left(\eta^{\prime}\right)\right),
$$

then the equilibrium fails the Intuitive Criterion.

In words, $S\left(\eta^{\prime}\right)$ consists of all types whose expected utility from choosing the in-equilibrium order quantity, $\eta$, is strictly greater than their maximum possible utility from making an out-ofequilibrium order quantity decision, $\eta^{\prime} \neq \eta$, over the set of best responses available to the equity holder. The equilibrium fails the Intuitive Criterion if there is a firm type not in $S\left(\eta^{\prime}\right)$ for which the utility from the equilibrium order quantity is less than the minimum possible utility that can be achieved by deviating from $\eta$ to $\eta^{\prime}$ given the equity holder's set of best responses.

Definition 4. Undefeated Refinement. As in (Mailath et al. 1993), we utilize some additional notation to present the Undefeated refinement for ease of exposition. A PBE is represented as a triplet of the form, $\psi=\left(\sigma_{N}, \sigma_{E}, \lambda\right)$, and, with a slight abuse of notation, the utility of a type $\tau$ firm relative to a particular PBE, $\psi$, is represented as $U_{N}(\tau, \psi)$. We introduce the Undefeated refinement by considering two equilibria, $\psi=\left(\sigma_{N}, \sigma_{E}, \lambda\right)$ and $\psi^{\prime}=\left(\sigma_{N}^{\prime}, \sigma_{E}^{\prime}, \lambda^{\prime}\right)$. The Undefeated refinement is applied by iterating the following process across all of the equilibria in the model.

The PBE, $\psi$, defeats the PBE, $\psi^{\prime}$, if there exists a stocking quantity, $\eta$, such that the following three conditions are satisfied:

1. $\forall \tau \in T: \sigma_{N}^{\prime} \neq \eta$ and $K \equiv\left\{\tau \in T \mid \sigma_{N}=\eta\right\} \neq \emptyset$;

2. $\forall \tau \in K: U_{N}(\tau, \psi) \geq U_{N}\left(\tau, \psi^{\prime}\right)$ and $\exists \tau \in K: U_{N}(\tau, \psi)>U_{N}\left(\tau, \psi^{\prime}\right)$; and

3. $\exists \tau \in K: \lambda^{\prime}(\tau \mid \eta) \neq \frac{g(\tau) \zeta(\tau)}{\sum_{\tilde{\tau} \in T} g(\tilde{\tau}) \zeta(\tilde{\tau})}$ for any $\zeta: T \rightarrow[0,1]$ satisfying

(a) $\tilde{\tau} \in K$ and $U_{N}(\tilde{\tau}, \psi)>U_{N}\left(\tilde{\tau}, \psi^{\prime}\right)$ implies $\zeta(\tilde{\tau})=1$,

(b) $\tilde{\tau} \notin K$ implies $\zeta(\tilde{\tau})=0$, and

(c) $\tilde{\tau} \in K$ and $U_{N}(\tilde{\tau}, \psi)=U_{N}\left(\tilde{\tau}, \psi^{\prime}\right)$ implies $\zeta(\tilde{\tau}) \in[0,1]$. 
In words, condition 1 states that $\psi^{\prime}$ must have an out-of-equilibrium stocking quantity choice that is an in-equilibrium stocking quantity choice in $\psi$. Condition 2 states that in $\psi$, an inequilibrium stocking quantity must be chosen by a set of types that prefers (strictly prefers for at least one type) their utility under $\psi$ compared to $\psi^{\prime}$. Condition 3 checks whether the out-ofequilibrium beliefs used to sustain $\psi^{\prime}$ are reasonable in light of $\psi$. The reasonableness of the beliefs a in $\psi^{\prime}$ re checked by assigning for each firm type a probability, $\zeta(\tilde{\tau})$, that the firm type chooses the out-of-equilibrium stocking quantity $\eta$. These probabilities are based on how the firm type behaves under $\psi$, so a probability of 1 is used if the firm type prefers the utility from $\eta$ under $\psi$ to the utility from $\eta^{\prime}$ under $\psi^{\prime}$, a probability of 0 is used if the firm type does not choose $\eta$ under $\psi$, and any probability may be used if the firm type is indifferent between the utility from $\eta$ under $\psi$ and the utility from $\eta^{\prime}$ under $\psi^{\prime}$.

\subsection{Proofs}

Proof of Lemma 1. We first show the existence of $\eta^{s}$. From (4), we get the type $\tau_{L}$ firm's utility function under high valuation as $U_{N}\left(\tau_{L}, \eta, \rho\left(\eta \mid \lambda\left(\tau_{H} \mid \eta\right)=1\right)\right)=(1-\alpha) \pi\left(\tau_{L}, \eta\right)+\alpha \pi\left(\tau_{H}, \eta\right)$ and under low valuation as $U_{N}\left(\tau_{L}, \eta, \rho\left(\eta \mid \lambda\left(\tau_{L} \mid \eta\right)=1\right)\right)=\pi\left(\tau_{L}, \eta\right)$. Both functions are concave, bounded from above, and tend to $-\infty$ as $\eta$ increases. The former function first order stochastically dominates the latter. Thus, it reaches its maximum for some $\eta \geq \eta_{L}^{*}$ and then decreases to $-\infty$. This implies that there exist values of $\eta>\eta_{L}^{*}$ such that $U_{N}\left(\tau_{L}, \eta, \rho\left(\eta \mid \lambda\left(\tau_{H} \mid \eta\right)=1\right)\right)<U_{N}\left(\tau_{L}, \eta_{L}^{*}, \rho\left(\eta_{L}^{*} \mid \lambda\left(\tau_{L} \mid \eta_{L}^{*}\right)=1\right)\right)$. The minimum order quantity over this set is $\eta^{s}$.

We now apply Definition 2 to actions $\eta \geq \eta^{s}$ for the type $\tau_{L}$ firm. For this, we set $\eta^{\prime}=\eta_{L}^{*}$ and show that actions $\eta \geq \eta^{s}$ are dominated by $\eta_{L}^{*}$ for the type $\tau_{L}$ firm. From inequality (5) in Definition 2, we need to show that

$$
\max _{\rho(\eta) \in P^{*}(T, \eta)} U_{N}\left(\tau_{L}, \eta, \rho(\eta)\right)<\min _{\rho\left(\eta_{L}^{*}\right) \in P^{*}\left(T, \eta_{L}^{*}\right)} U_{N}\left(\tau_{L}, \eta_{L}^{*}, \rho\left(\eta_{L}^{*}\right)\right) .
$$

First order stochastic dominance and concavity of the utility function imply that the utility for the type $\tau_{L}$ firm, $U_{N}\left(\tau_{L}, \eta, \rho(\eta)\right)$, is maximized with respect to $\rho(\eta)$ when $\lambda\left(\tau_{L} \mid \eta\right)=0$ and is minimized when $\lambda\left(\tau_{L} \mid \eta\right)=1$. Substituting these posterior beliefs into the utility function and using (4), for 
any $\eta \geq \eta^{s}$ we get

$$
\begin{aligned}
\max _{\rho(\eta) \in P^{*}(T, \eta(\eta))} U_{N}\left(\tau_{L}, \eta, \rho\right) & =(1-\alpha) \pi\left(\tau_{L}, \eta\right)+\alpha \pi\left(\tau_{H}, \eta\right) \\
& \leq(1-\alpha) \pi\left(\tau_{L}, \eta^{s}\right)+\alpha \pi\left(\tau_{H}, \eta^{s}\right) \\
& <\pi\left(\tau_{L}, \eta_{L}^{*}\right) \\
& =\min _{\rho\left(\eta_{L}^{*}\right) \in P^{*}\left(T, \eta_{L}^{*}\right)} U_{N}\left(\tau_{L}, \eta_{L}^{*}, \rho\left(\eta_{L}^{*}\right)\right) .
\end{aligned}
$$

Here, the first inequality follows because $(1-\alpha) \pi\left(\tau_{L}, \eta\right)+\alpha \pi\left(\tau_{H}, \eta\right)$ reaches its maximum at an order quantity less than $\eta^{s}$ and is decreasing in $\eta$ for $\eta \geq \eta^{s}$. The second inequality follows from the definition of $\eta^{s}$.

According to the reasonable beliefs refinement in Definition 2, a PBE has reasonable beliefs if those beliefs put zero probability that a signal which is strictly dominated for a type $\tau_{i}$ firm was sent by a type $\tau_{i}$ firm, i.e. $\lambda\left(\tau_{i} \mid \eta\right)=0$. For a type $\tau_{L}$ firm, since any $\eta \geq \eta^{s}$ meets the definition of strict dominance, the equity holder's beliefs should place zero probability that such a signal was sent by a type $\tau_{L}$ firm. Moreover, the definition of $\eta^{s}$ implies that no order quantity less than $\eta^{s}$ is strictly dominated for a type $\tau_{L}$ firm. This proves the lemma.

We require the following lemma for the subsequent proofs.

Lemma 2. For firm types $\tau_{H}$ and $\tau_{H}$ for which $F_{\tau_{H}}$ FOSD $F_{\tau_{L}}$, the following properties hold:

1. $\pi\left(\tau_{H}, \eta\right) \geq \pi\left(\tau_{L}, \eta\right)$ for all $\eta$ and $\pi\left(\tau_{H}, \eta\right)>\pi\left(\tau_{L}, \eta\right)$ for some $\eta$.

2. $\pi\left(\tau_{H}, \eta+Q\right)-\pi\left(\tau_{H}, \eta\right) \geq \pi\left(\tau_{L}, \eta+Q\right)-\pi\left(\tau_{L}, \eta\right)$ for all $\eta$ and $\pi\left(\tau_{H}, \eta+Q\right)-\pi\left(\tau_{H}, \eta\right)>$ $\pi\left(\tau_{L}, \eta+Q\right)-\pi\left(\tau_{L}, \eta\right)$ for some $\eta$.

Proof. Omitted.

Proof of Proposition 1. We show that the three conditions stated in the proposition are sufficient for a pooling PBE at $\eta^{p}$. For this, we solve for the best response functions of the firm and the equity holder under the specified posterior beliefs of the equity holder. The best response function of the equity holder follows from (3) for all values of $\eta$ and $\lambda(\tau \mid \eta)$.

If $\eta_{H}^{*}<\eta^{s}$, then the posterior beliefs of the equity holder are well-defined. To see this, note that $\eta^{p}<\eta_{H}^{*}$ by first order stochastic dominance. Thus, $\eta^{p}<\eta_{H}^{*}$ and $\eta_{H}^{*}<\eta^{s}$ together imply that $\eta^{p}<\eta^{s}$. 
We now confirm that the proposed equilibrium maximizes the utility of each firm type so that no firm type has an incentive to deviate. We must show that $\eta^{p}=\arg \max _{\eta} U_{N}\left(\tau_{j}, \eta, \rho^{*}(\eta)\right)$ for $j=L, H$ across the three intervals defined by the posterior beliefs of the equity holder, namely $\eta<\eta^{p}, \eta^{p} \leq \eta<\eta^{s}$, and $\eta \geq \eta^{s}$.

Consider first the type $\tau_{L}$ firm. The expected utility of the firm is given by (4) as

$$
U_{N}\left(\tau_{L}, \eta, \rho^{*}(\eta)\right)= \begin{cases}\pi\left(\tau_{L}, \eta\right) & \text { for } \eta<\eta^{p}, \\ \left(1-\alpha+\alpha g\left(\tau_{L}\right)\right) \pi\left(\tau_{L}, \eta\right)+\alpha g\left(\tau_{H}\right) \pi\left(\tau_{H}, \eta\right) & \text { for } \eta^{p} \leq \eta<\eta^{s}, \\ (1-\alpha) \pi\left(\tau_{L}, \eta\right)+\alpha \pi\left(\tau_{H}, \eta\right) & \text { for } \eta \geq \eta^{s} .\end{cases}
$$

We have three cases. (i) A type $\tau_{L}$ firm does not deviate from $\eta^{p}$ to any $\eta<\eta^{p}$ if $U_{N}\left(\tau_{L}, \eta^{p}, \rho^{*}\left(\eta^{p}\right)\right)>$ $\max _{\eta<\eta^{p}} U_{N}\left(\tau_{L}, \eta, \rho^{*}(\eta)\right)$, i.e., if $U_{N}\left(\tau_{L}, \eta^{p}, \rho^{*}\left(\eta^{p}\right)\right)>U_{N}\left(\tau_{L}, \eta_{L}^{*}, \rho^{*}\left(\eta_{L}^{*}\right)\right)$. This gives inequality (8) as a sufficient condition in the proposition. (ii) In order to ensure that a type $\tau_{L}$ firm does not deviate from $\eta^{p}$ to any $\eta^{p}<\eta<\eta^{s}$, it must be true that $U_{N}\left(\tau_{L}, \eta^{p}, \rho^{*}\left(\eta^{p}\right)\right) \geq \max _{\eta^{p} \leq \eta<\eta^{s}} U_{N}\left(\tau_{L}, \eta, \rho^{*}(\eta)\right)$. This condition holds because $\eta^{p}$ maximizes the expected utility of the type $\tau_{H}$ firm under weighted valuation, which implies by first order stochastic dominance that the maximizer of the expected utility of the type $\tau_{L}$ firm under weighted valuation is less than or equal to $\eta^{p}$, and therefore, the expected utility of the type $\tau_{L}$ firm is decreasing in $\eta$ in the interval $\eta^{p}<\eta<\eta^{s}$. (iii) Finally, a type $\tau_{L}$ firm does not deviate from $\eta^{p}$ to any $\eta \geq \eta^{s}$ by the definition of $\eta^{s}$. By Lemma 1 , the type $\tau_{L}$ firm receives a higher expected utility by stocking $\eta_{L}^{*}$ than by selecting any stocking quantity $\eta \geq \eta^{s}$. This combined with the condition that $U_{N}\left(\tau_{L}, \eta^{p}, \rho^{*}\left(\eta^{p}\right)\right)>U_{N}\left(\tau_{L}, \eta_{L}^{*}, \rho^{*}\left(\eta_{L}^{*}\right)\right)$ precludes a deviation to any $\eta \geq \eta^{s}$ by a type $\tau_{L}$ firm.

Now consider the type $\tau_{H}$ firm. Her expected utility is also given by (4) as

$$
U_{N}\left(\tau_{H}, \eta, \rho^{*}(\eta)\right)= \begin{cases}\alpha \pi\left(\tau_{L}, \eta\right)+(1-\alpha) \pi\left(\tau_{H}, \eta\right) & \text { for } \eta<\eta^{p}, \\ \alpha g\left(\tau_{L}\right) \pi\left(\tau_{L}, \eta\right)+\left(1-\alpha+\alpha g\left(\tau_{H}\right)\right) \pi\left(\tau_{H}, \eta\right) & \text { for } \eta^{p} \leq \eta<\eta^{s} \\ \pi\left(\tau_{H}, \eta\right) & \text { for } \eta \geq \eta^{s} .\end{cases}
$$

Again, we have three cases. (i) Note that the expected utility of the firm for $\eta<\eta^{p}$ is computed under low valuation. Its value is less than the corresponding utility under weighted valuation. Moreover, $\eta^{p}$ maximizes the expected utility of the type $\tau_{H}$ firm under weighted valuation. Therefore, a type $\tau_{H}$ firm receives a higher utility by stocking $\eta^{p}$ than any $\eta<\eta^{p}$. Thus, she does not deviate from $\eta^{p}$ to any $\eta<\eta^{p}$. (ii) A type $\tau_{H}$ firm also does not deviate from $\eta^{p}$ to any $\eta^{p}<\eta<\eta^{s}$ because by definition $\eta^{p}$ maximizes the expected utility of the 
type $\tau_{H}$ firm in this interval. (iii) Finally, in order to ensure that a type $\tau_{H}$ firm does not deviate from $\eta^{p}$ to some $\eta \geq \eta^{s}$, it must be that $U_{N}\left(\tau_{H}, \eta^{p}, \rho^{*}\left(\eta^{p}\right)\right)>\max _{\eta \geq \eta^{s}} U_{N}\left(\tau_{H}, \eta, \rho^{*}(\eta)\right)$. If $\eta_{H}^{*}<\eta^{s}$, then the maximum on the right hand side of the inequality is achieved at $\eta^{s}$. Thus, this requirement can be simplified to $U_{N}\left(\tau_{H}, \eta^{p}, \rho^{*}\left(\eta^{p}\right)\right)>U_{N}\left(\tau_{H}, \eta^{s}, \rho^{*}\left(\eta^{s}\right)\right)$. Thus, $\eta_{H}^{*}<\eta^{s}$ and $U_{N}\left(\tau_{H}, \eta^{p}, \rho^{*}\left(\eta^{p}\right)\right)>U_{N}\left(\tau_{H}, \eta^{s}, \rho^{*}\left(\eta^{s}\right)\right)$ are sufficient conditions for $\eta^{p}$ to be the order quantity that maximizes the expected utility of the type $\tau_{H}$ firm.

Thus, the conditions specified in (7), (8), and (9) are sufficient to show a pooling equilibrium at $\eta^{p}$.

\section{Proof of Proposition 2.}

In the separating $\mathrm{PBE}$, a type $\tau_{H}$ firm chooses the least cost separating stocking quantity, $\eta=\max \left(\eta^{s}, \eta_{H}^{*}\right)$, and receives a high valuation while a type $\tau_{L}$ firm chooses $\eta=\eta_{L}^{*}$ and receives a low valuation. When $\eta^{s} \leq \eta_{H}^{*}$ it will always result in a separating PBE in which the type $\tau_{H}$ firm chooses stocking quantity $\eta_{H}^{*}$ as doing so will maximize both her short-term valuation and expected long-term profits. In this case, the type $\tau_{L}$ firm's type is revealed since, by strict dominance, she is unwilling to select a stocking quantity that is $\eta \geq \eta^{s}$. The type $\tau_{L}$ firm therefore optimizes her utility by choosing $\eta_{L}^{*}$.

In the case where $\eta^{s}>\eta_{H}^{*}$, a separating PBE under reasonable beliefs is precluded if the equilibrium stocking quantity for either type is strictly dominated by any alternative stocking quantity. Recall that a stocking quantity is strictly dominated if Inequality (5) is true. We evaluate whether Inequality (5) holds for either firm type. Consider first a type $\tau_{L}$ firm. The inequality simplifies to $U_{N}\left(\tau_{L}, \eta_{L}^{*}, \rho^{*}\left(\eta_{L}^{*}\right)\right)<\max _{\eta^{\prime} \neq \eta_{L}^{*}} U_{N}\left(\tau_{L}, \eta^{\prime}, \rho\left(\eta^{\prime} \mid \lambda\left(\tau_{L} \mid \eta^{\prime}\right)=1\right)\right)$. This is not true for any value of $\eta^{\prime}$.

For a type $\tau_{H}$ firm, Inequality (5) is $U_{N}\left(\tau_{H}, \eta^{s}, \rho^{*}\left(\eta^{s}\right)\right)<\max _{\eta^{\prime} \neq \eta^{s}} U_{N}\left(\tau_{H}, \eta^{\prime}, \rho\left(\eta^{\prime} \mid \lambda\left(\tau_{L} \mid \eta^{\prime}\right)=1\right)\right)$. There may be conditions under which this is true, and therefore $\eta^{s}$ is strictly dominated for type $\tau_{H}$ firm. This implies that when $\eta_{H}^{*}<\eta^{s}$, Inequality (12) must hold for a separating PBE to exist under reasonable beliefs.

Proof of Proposition 3. We prove that the conditions identified in Proposition 3 are necessary and sufficient for the pooling PBE from Proposition 1 to survive the Intuitive Criterion. We do this by showing that these conditions are equivalent to the conditions identified in the definition of the Intuitive Criterion refinement in the case of a pooling PBE at $\eta^{p}$. The Intuitive Criterion 
refinement is defined in Cho and Kreps (1987) and summarized using our notation in Definition 3. To evaluate the pooling PBE defined in Proposition 1 using the Intuitive Criterion refinement, form the set $S\left(\eta^{\prime}\right)$ for all $\eta^{\prime} \neq \eta^{p}$ consisting of all types, $\tau$, such that

$$
U_{N}\left(\tau, \eta^{p}, \rho^{*}\left(\eta^{p}\right)\right)>\max _{\rho\left(\eta^{\prime}\right) \in P^{*}\left(T, \eta^{\prime}\right)} U_{N}\left(\tau, \eta^{\prime}, \rho\left(\eta^{\prime}\right)\right) .
$$

The equilibrium fails the Intuitive Criterion if there exists some type $\tau^{\prime} \in T$ and $\tau^{\prime} \notin S\left(\eta^{\prime}\right)$ such that

$$
U_{N}\left(\tau^{\prime}, \eta^{p}, \rho^{*}\left(\eta^{p}\right)\right)<\min _{\rho\left(\eta^{\prime}\right) \in P^{*}\left(T \backslash S(\eta), \eta^{\prime}\right)} U_{N}\left(\tau^{\prime}, \eta^{\prime}, \rho\left(\eta^{\prime}\right)\right)
$$

To apply the Intuitive Criterion, there are two ranges of $\eta^{\prime}$ that we must evaluate, $\eta^{\prime}<\eta^{p}$ and $\eta^{\prime}>\eta^{p}$. We first consider a deviation to $\eta^{\prime}<\eta^{p}$. Recall that the utility function of a type $\tau$ firm is $U_{N}\left(\tau, \eta^{\prime}, \rho\left(\eta^{\prime}\right)\right)=\alpha \rho\left(\eta^{\prime}\right)+\{1-\alpha\} \pi\left(\tau, \eta^{\prime}\right)$. Using the result from Lemma 2 that $\frac{\Delta \pi\left(\tau_{H}, \eta\right)}{\Delta \eta} \geq \frac{\Delta \pi\left(\tau_{L}, \eta\right)}{\Delta \eta}$, any deviation to $\eta^{\prime}<\eta^{p}$ that yields in a higher utility for a type $\tau_{H}$ firm will also yield in a higher utility for a type $\tau_{L}$ firm. Therefore by (13), for any $\eta^{\prime}<\eta^{p}, S\left(\eta^{\prime}\right)=\emptyset$, $S\left(\eta^{\prime}\right)=\tau_{H}$ or $S\left(\eta^{\prime}\right)=T$. From (14), the Intuitive Criterion will not eliminate the equilibrium with a value of $\eta^{\prime}$ if $S\left(\eta^{\prime}\right)=\emptyset$ or $S\left(\eta^{\prime}\right)=T$. If the value of $\eta^{\prime}$ is such that $S\left(\eta^{\prime}\right)=\tau_{H}$, then Inequality (14) can be expressed as $U_{N}\left(\tau_{L}, \eta^{p}, \rho^{*}\left(\eta^{p}\right)\right)<U_{N}\left(\tau_{L}, \eta^{\prime}, \rho\left(\eta^{\prime} \mid \lambda\left(\tau_{L} \mid \eta^{\prime}\right)=1\right)\right)$. However, this inequality cannot be true for a pooling PBE at $\eta^{p}$ since the existence of a pooling PBE at $\eta^{p}$ already requires that $U_{N}\left(\tau_{L}, \eta^{p}, \rho^{*}\left(\eta^{p}\right)\right)>U_{N}\left(\tau_{L}, \eta_{L}^{*}, \rho^{*}\left(\eta_{L}^{*}\right)\right)$.

We next consider a deviation to $\eta^{\prime}>\eta^{p}$. Recalling again the form of the firm's utility function and the results of Lemma 2, any deviation to $\eta^{\prime}>\eta^{p}$ that results in a higher utility for a type $\tau_{L}$ firm will also result in a higher utility for a type $\tau_{H}$ firm. Therefore, for any $\eta^{\prime}>\eta^{p}, S\left(\eta^{\prime}\right)=\emptyset$, $S\left(\eta^{\prime}\right)=\tau_{L}$ or $S\left(\eta^{\prime}\right)=T$. Again from (14), the Intuitive Criterion will not eliminate the equilibrium with a value of $\eta^{\prime}$ if $S\left(\eta^{\prime}\right)=\emptyset$ or $S\left(\eta^{\prime}\right)=T$. If there exists some $\eta^{\prime}$ such that $S\left(\eta^{\prime}\right)=\tau_{L}$, then inequality (13) could be expressed as

$$
U_{N}\left(\tau_{L}, \eta^{p}, \rho^{*}\left(\eta^{p}\right)\right)>U_{N}\left(\tau_{L}, \eta^{\prime}, \rho\left(\eta^{\prime} \mid \lambda\left(\tau_{H} \mid \eta^{\prime}\right)=1\right)\right)
$$

and inequality (14) could be expressed as

$$
U_{N}\left(\tau_{H}, \eta^{p}, \rho^{*}\left(\eta^{p}\right)\right)<U_{N}\left(\tau_{H}, \eta^{\prime}, \rho\left(\eta^{\prime} \mid \lambda\left(\tau_{H} \mid \eta^{\prime}\right)=1\right)\right)
$$

By the definition of the Intuitive Criterion refinement, the pooling PBE identified in Proposition 1 will survive the Intuitive Criterion refinement if and only if there does not exist a stocking quantity for which both of these conditions are true. 
Proof of Proposition 4. We seek to prove that if one or more pooling PBE exists under reasonable beliefs, then at least one of those PBE will survive the Undefeated refinement but no separating PBE will.

Let $Z$ represent the set of all stocking quantities at which there is a pooling PBE (based on Corollary 1). Let $\eta^{L p}$ be the maximum stocking quantity at the pooling PBE within this set which maximizes the utility of the type $\tau_{L}$ firm and let $\eta^{H p}$ be the minimum stocking quantity at the pooling PBE within this set which maximizes the utility of the type $\tau_{H}$ firm,

$$
\begin{aligned}
\eta^{L p} & =\max \left\{\eta: \underset{\eta \in Z}{\arg \max } U\left(\tau_{L}, \eta, \rho\left(\eta \mid \lambda\left(\tau_{L} \mid \eta\right)=g\left(\tau_{L}\right)\right)\right)\right\}, \\
\eta^{H p} & =\min \left\{\eta: \underset{\eta \in Z}{\arg \max } U\left(\tau_{H}, \eta, \rho\left(\eta \mid \lambda\left(\tau_{H} \mid \eta\right)=g\left(\tau_{H}\right)\right)\right)\right\} .
\end{aligned}
$$

Any pooling PBE in $Z$ defeats the least cost separating PBE (if one exists based on Proposition 2). This is from the definition of the Undefeated refinement and because a pooling PBE based on Corollary 1 only exists if it provides a utility greater than the separating PBE for both firm types. Furthermore, from the concavity of the utility functions, the pooling PBE at $\eta^{L p}$ defeats any pooling PBE at $\eta<\eta^{L p}$ and the pooling PBE at $\eta^{H p}$ defeats any pooling PBE at $\eta>\eta^{H p}$.

By FOSD, $\eta^{L p} \leq \eta^{H p}$. If $\eta^{L p}=\eta^{H p}$ then the pooling PBE at this stocking quantity is the unique undefeated PBE since there is no other PBE which provides at least the same utility for both firm types and a higher utility for at least one of the firm types. If $\eta^{L p}<\eta^{H p}$ then the pooling PBEs at all $\eta \in\left[\eta^{L p}, \eta^{H p}\right]$ are undefeated. This is from the concavity of the utility functions, which implies that for all of the pooling PBEs at stocking quantities $\eta \in\left[\eta^{L p}, \eta^{H p}\right]$, no other PBE exists which provides at least the same utility for both firm types and a higher utility for at least one of the firm types. 
Figures 1a and 1b. Utility functions for a $\tau_{\mathrm{L}}$ type firm under the low, weighted and high valuation, and for a $\tau_{\mathrm{H}}$ type firm under the weighted and high valuation. The model parameters are: $\alpha=0.80, g\left(\tau_{L}\right)=0.20$, demand follows an exponential distribution with $\mu_{L}=500$ and $\mu_{H}=800, p=1.00, c=0.60, s=0.00, Q=50$.

Figure 1a. Firm utility functions showing a pooling PBE at $\eta^{p}=400$.

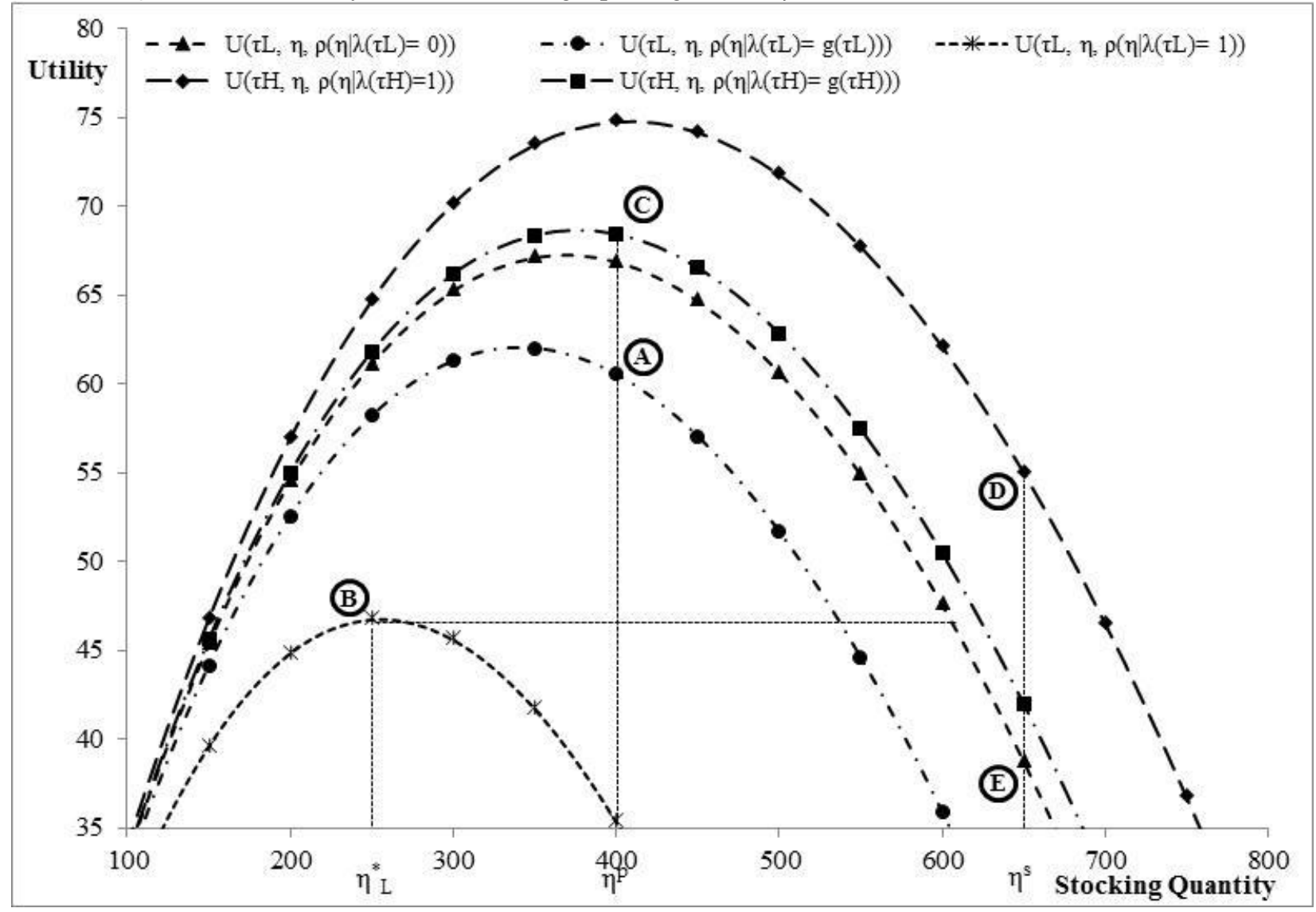


Figure 1b. Firm utility functions showing that the pooling PBE at $\eta^{p}=400$ survives the Intuitive Criterion refinement.

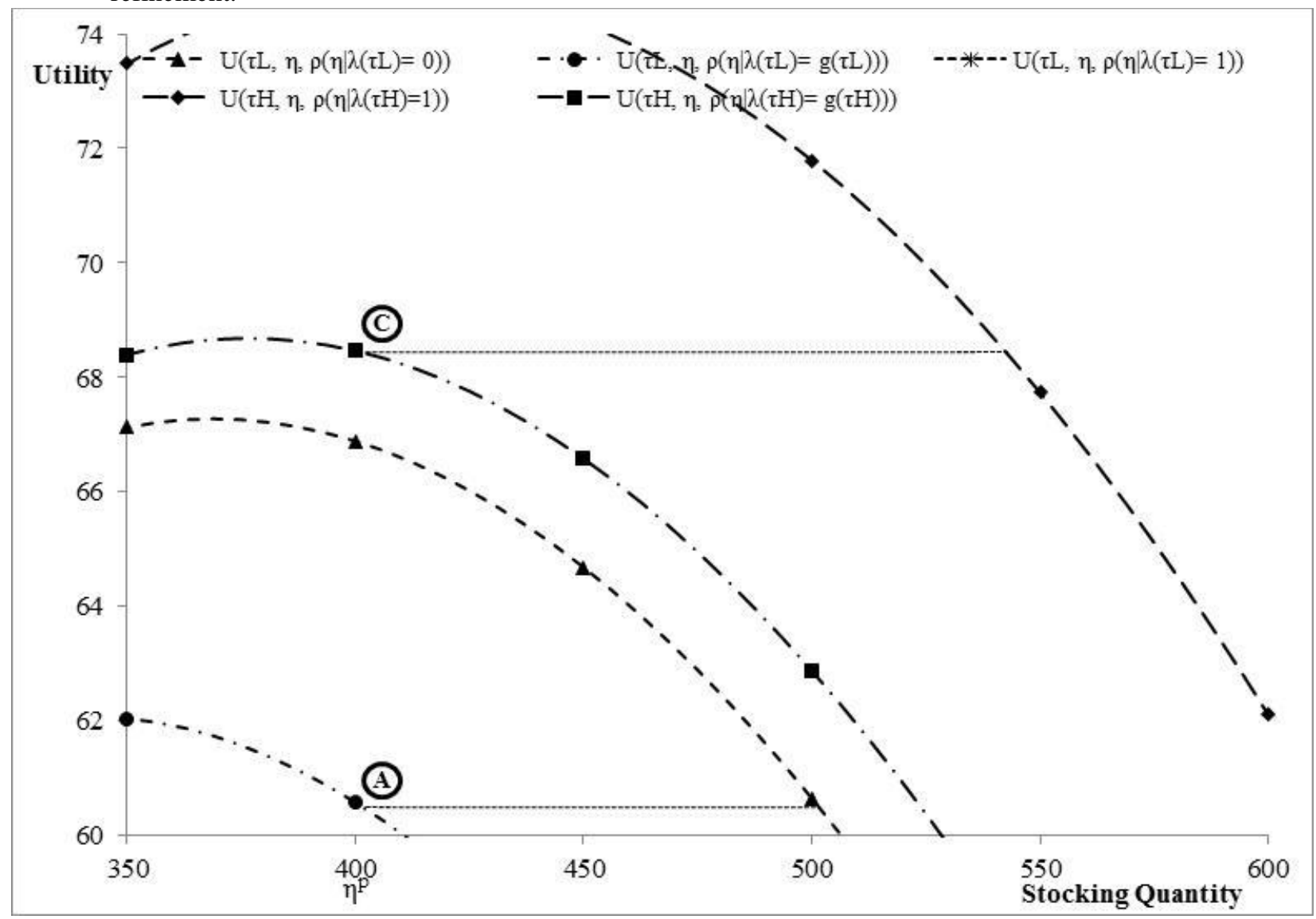


Figure 2. An example of when a separating PBE does not exist. For this example, the model parameters are: $\alpha=$ $0.80, g\left(\tau_{L}\right)=0.20$, demand follows an exponential distribution with $\mu_{L}=500$ and $\mu_{H}=800, p=1.00, c=0.60, s=$ $0.00, Q=100$.

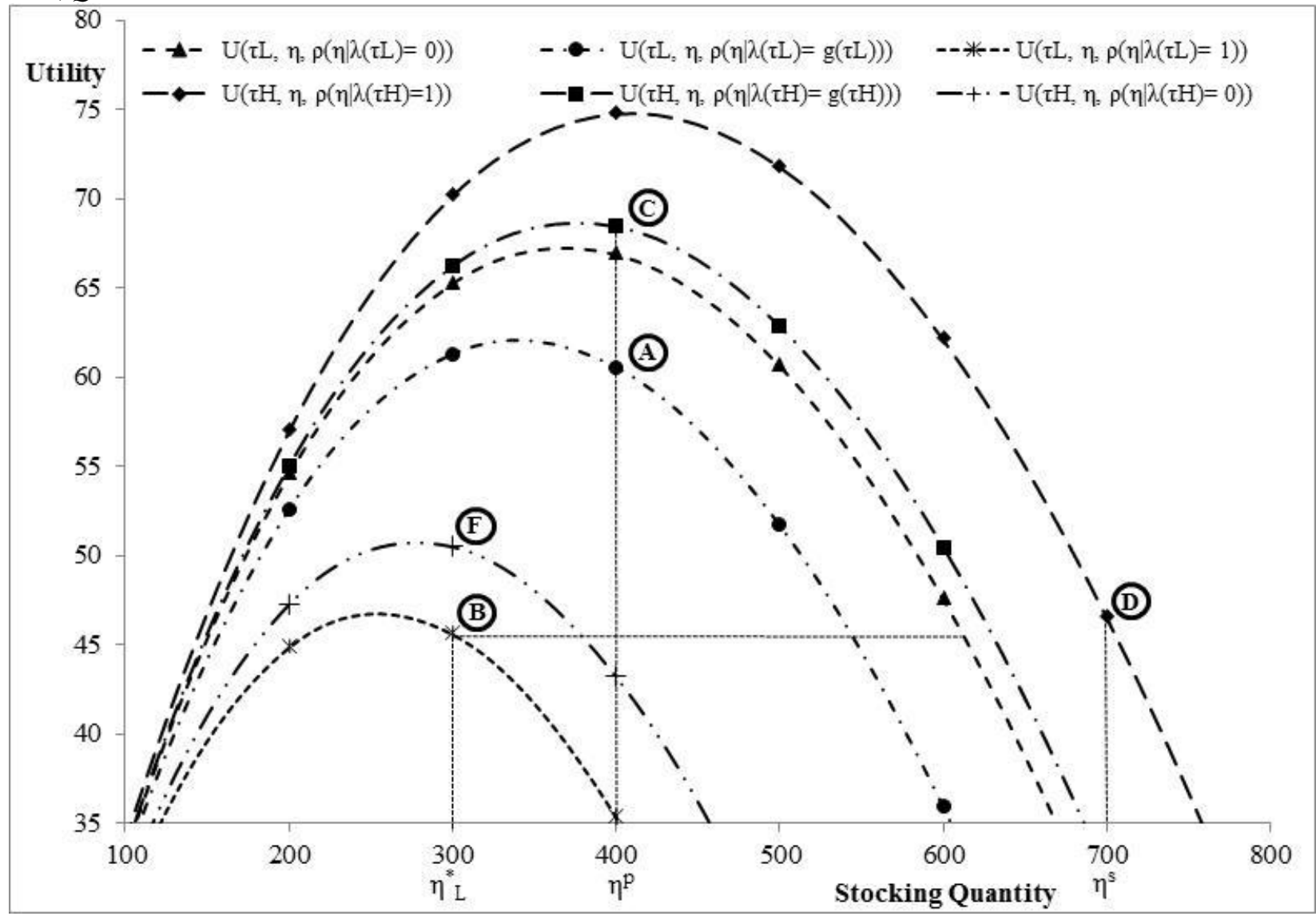


Figure 3a, 3b and 3c. Each figure shows the PBE outcomes across a range of values for $\alpha$ and $g\left(\tau_{L}\right)$. Dark grey boxes identify scenarios for which a pooling PBE at $\eta^{p}$ survives the Intuitive Criterion refinement. Medium grey boxes identify scenarios for which a pooling PBE at $\eta^{p}$ does not survive the Intuitive Criterion refinement. Light grey boxes identify scenarios for which there is not a pooling PBE at $\eta^{p}$.

Figure 3a. The model parameters are: demand follows an exponential distribution with $\mu_{L}=500$ and $\mu_{H}=1000$, $p=1.00, c=0.20, s=0.00, Q=100$.

Short Term-ism, $\alpha$

$\begin{array}{llllllllllllllllllll}0.05 & 0.10 & 0.15 & 0.20 & 0.25 & 0.30 & 0.35 & 0.40 & 0.45 & 0.50 & 0.55 & 0.60 & 0.65 & 0.70 & 0.75 & 0.80 & 0.85 & 0.90 & 0.95 & 1.00\end{array}$

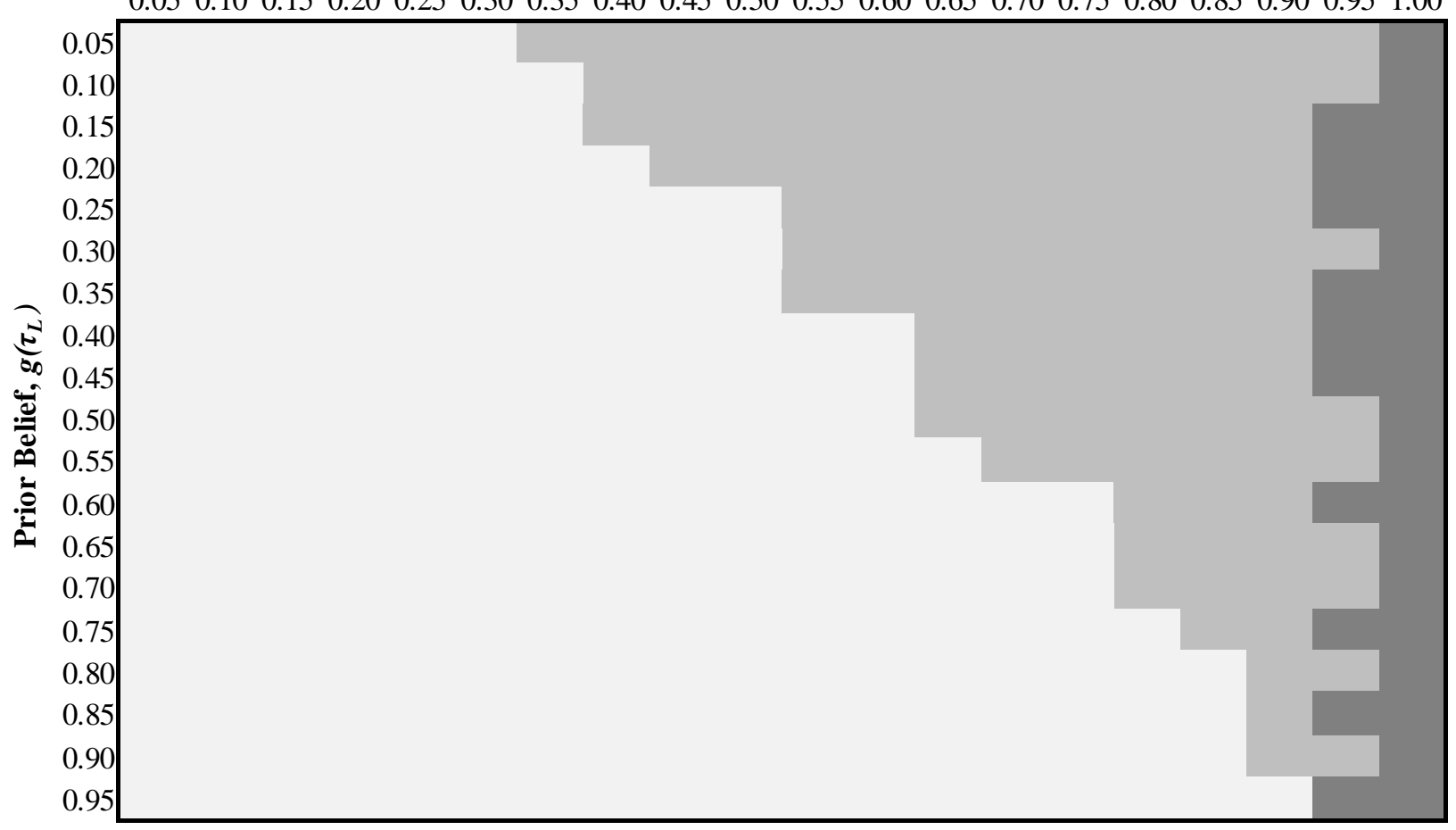


Figure 3b. The model parameters are: demand follows an exponential distribution with with $\mu_{L}=500$ and $\mu_{H}=$ $800, p=1.00, c=0.60, s=0.00, Q=50$.

\section{Short Term-ism, $\alpha$}

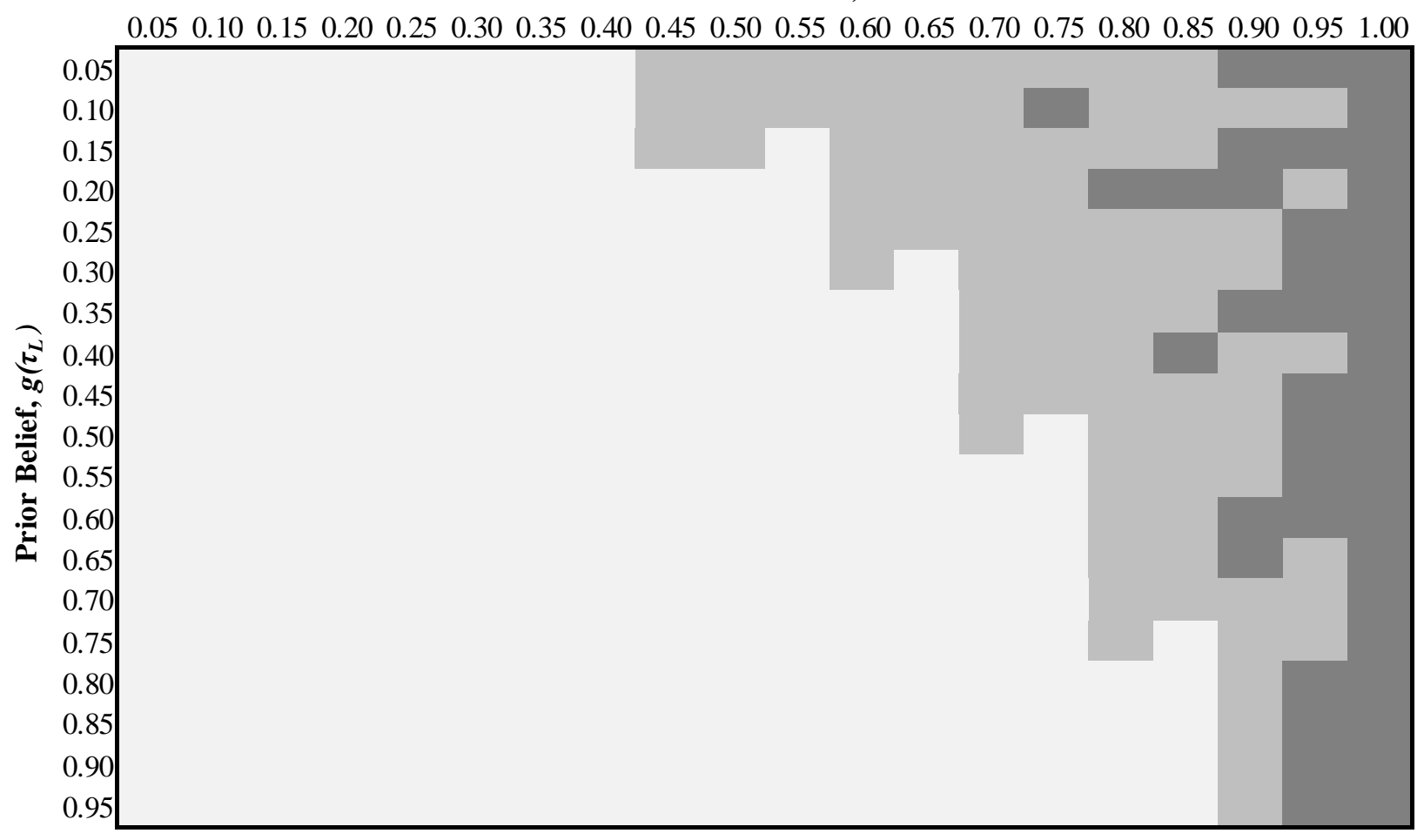

Figure 3c. The model parameters are: demand follows an exponential distribution with $\mu_{L}=500$ and $\mu_{H}=600, p$ $=1.00, c=0.80, s=0.00, Q=10$.

Short Term-ism, $\alpha$

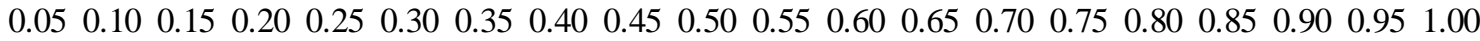

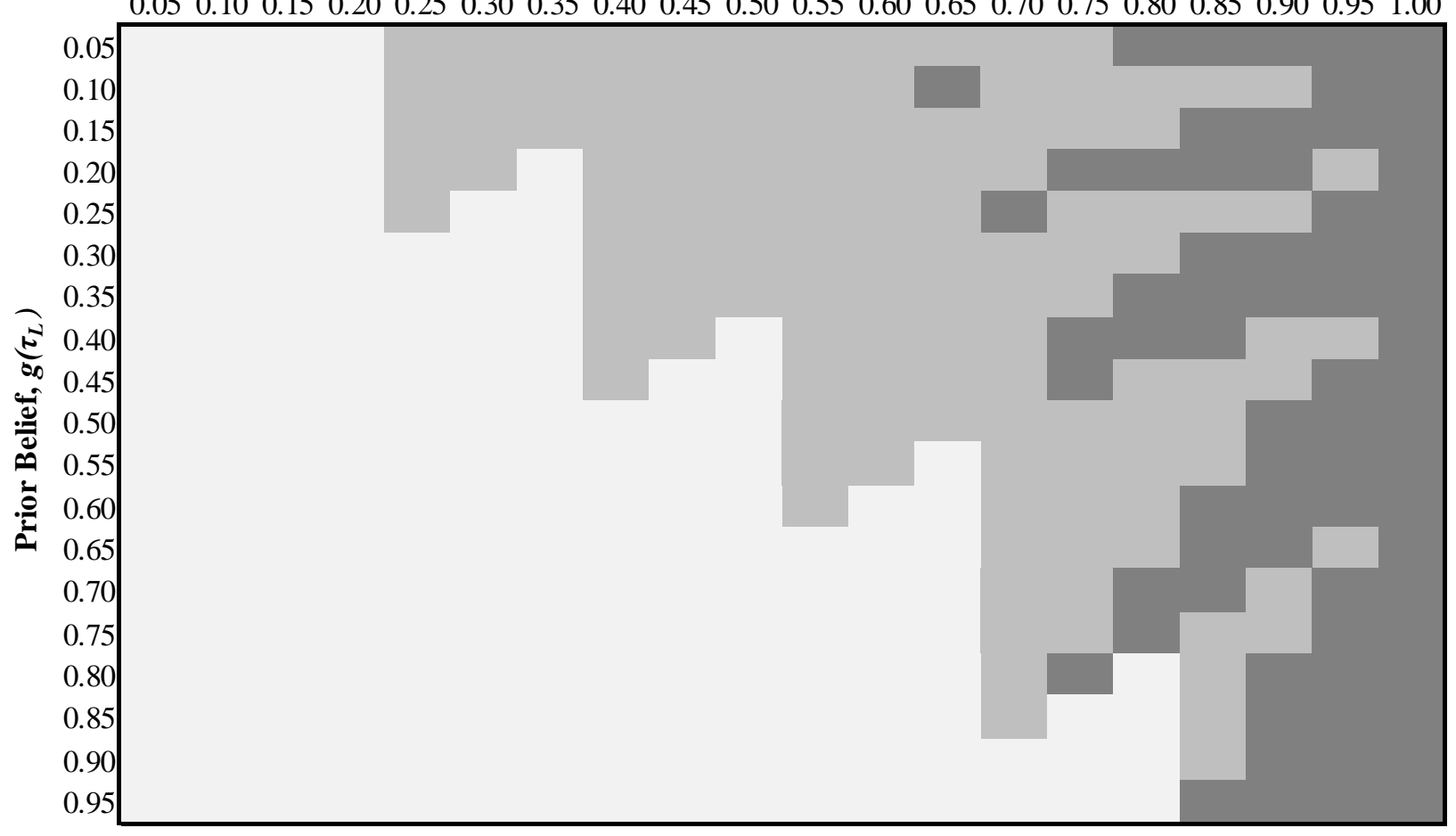

